\title{
Reversible cardiac disease features in an inducible CUG repeat RNA-expressing mouse model of myotonic dystrophy
}

\author{
Ashish N. Rao, ${ }^{1}$ Hannah M. Campbell, ${ }^{2,3}$ Xiangnan Guan, ${ }^{4}$ Tarah A. Word, ${ }^{2}$ Xander H.T. Wehrens,,${ }^{2,5}$ \\ Zheng Xia, ${ }^{4,6}$ and Thomas A. Cooper ${ }^{1,2,5,7}$ \\ 'Department of Molecular and Cellular Biology, ${ }^{2}$ Department of Molecular Physiology and Biophysics, and ${ }^{3}$ Medical \\ Scientist Training Program, Baylor College of Medicine, Houston, Texas, USA. ${ }^{4}$ Computational Biology Program, Oregon \\ Health \& Science University, Portland, Oregon, USA. ${ }^{5}$ Cardiovascular Research Institute, Baylor College of Medicine, \\ Houston, Texas, USA. ${ }^{6}$ Department of Molecular Microbiology and Immunology, Oregon Health \& Science University, \\ Portland, Oregon, USA. 'Department of Pathology and Immunology, Baylor College of Medicine, Houston, Texas, USA.
}

\begin{abstract}
Myotonic dystrophy type 1 (DM1) is caused by a CTC repeat expansion in the DMPK gene. Expression of pathogenic expanded CUG repeat (CUGexp) RNA causes multisystemic disease by perturbing the functions of RNA-binding proteins, resulting in expression of fetal protein isoforms in adult tissues. Cardiac involvement affects $50 \%$ of individuals with DM1 and causes $25 \%$ of disease-related deaths. We developed a transgenic mouse model for tetracycline-inducible and heart-specific expression of human DMPK mRNA containing 960 CUG repeats. CUGexp RNA is expressed in atria and ventricles and induced mice exhibit electrophysiological and molecular features of DM1 disease, including cardiac conduction delays, supraventricular arrhythmias, nuclear RNA foci with Muscleblind protein colocalization, and alternative splicing defects. Importantly, these phenotypes were rescued upon loss of CUGexp RNA expression. Transcriptome analysis revealed gene expression and alternative splicing changes in ion transport genes that are associated with inherited cardiac conduction diseases, including a subset of genes involved in calcium handling. Consistent with RNA-Seq results, calcium-handling defects were identified in atrial cardiomyocytes isolated from mice expressing CUGexp RNA. These results identify potential tissue-specific mechanisms contributing to cardiac pathogenesis in DM1 and demonstrate the utility of reversible phenotypes in our model to facilitate development of targeted therapeutic approaches.
\end{abstract}

Conflict of interest: XHTW is a founding partner of Elex Biotech, a start-up company that develops drug molecules that target RyRs for the treatment of cardiac arrhythmia disorders.

Copyright: ( $) 2021$, Rao et al. This is an open access article published under the terms of the Creative Commons Attribution 4.0 International License.

Submitted: August 21, 2020

Accepted: January 20, 2021

Published: March 8, 2021

Reference information: /CI Insight. 2021;6(5):e143465.

https://doi.org/10.1172/jici.

insight.143465.

\section{Introduction}

Myotonic dystrophy type 1 (DM1) is the most common form of adult-onset muscular dystrophy characterized by multisystemic manifestations affecting skeletal muscle, cardiac, central nervous system, and gastrointestinal tissues (1). DM1 is an autosomal dominant disease caused by a CTG trinucleotide repeat expansion in the $3^{\prime}$ untranslated region of the $D M P K$ gene $(2,3)$. The gain-of-function expanded CUG repeat (CUGexp) RNA transcribed from the mutated allele is well established as the pathogenic basis for DM1 (4). CUGexp RNA transcripts retained in the nuclei in the form of foci $(5,6)$ disrupt the activities of the RNA-binding proteins Muscleblind-like (MBNL) $(7,8)$ and CUGBP, Elav-like family member (CELF) $(9$, $10)$, thereby perturbing various aspects of posttranscriptional gene regulation. The best-characterized effects are the disruption of developmentally regulated alternative splicing and polyadenylation programs (11-15).

Cardiac involvement is a prominent feature of DM1 and is the second leading cause of mortality in affected individuals, following respiratory insufficiency due to skeletal muscle wasting $(16,17)$. In various population studies in patients with adult-onset DM1, cardiac manifestations have been noted in patient groups with a median age of 40 years $(18,19)$. Recent reports have also described cardiac disease features in children with congenital DM1 $(20,21)$. The cardiac manifestations in DM1 are multifaceted and primarily consist of conduction delays and both supraventricular and ventricular arrhythmias. Conduction delays include prolongation of the PR interval, QRS complex, and QT interval (22-24). Individuals with DM1 exhibit a variety of arrhythmias, the more common of which are those of supraventricular origin, consisting of sinus node dysfunction, atrial flutter, atrial fibrillation, and supraventricular tachycardia $(17,22,25)$. Additionally, affected individuals present with ventricular arrhythmias such as premature ventricular contractions and ventricular tachycardia 
(24). Histological examination of DM1 heart samples showed fibrosis and fatty infiltration within the conduction system as well as myocyte hypertrophy $(26,27)$. Additionally, diffuse interstitial fibrosis in the myocardium is also observed (28). Apart from the more common effects on cardiac conduction, a small proportion of affected individuals also exhibit left ventricular hypertrophy, dilatation, and systolic dysfunction (29, 30).

The tissue-specific molecular mechanisms contributing to the complex cardiac phenotypes in DM1 are not well understood. Additionally, there have been a limited number of studies focused on the development of therapeutic strategies for targeting of CUGexp RNA in heart for treatment of cardiac DM1 disease features. Animal models have proven to be an excellent resource for interrogating various aspects of DM1 disease pathology. Mice engineered to model DM1-associated MBNL loss of function by a combined Mbnl1 and Mbnl2 knockout (31) or CELF1 gain of function by transgenic overexpression of human CELF1 protein (32) recapitulated several cardiac molecular and physiological disease features. Previously, CUGexp RNA-expressing mouse models of differing repeat lengths have displayed a variable range of DM1like phenotypes. The $\mathrm{CTG}_{5}$ and $\mathrm{CTG}_{200}$ models display alternative splicing changes, $\mathrm{PR}$ prolongation, and atrioventricular blocks $(33,34)$. The LC15 model expressing 200 CUG repeats exhibited prolongation of QRS and corrected QT (QTc) intervals and increased susceptibility to ventricular arrhythmias in response to flecainide (class Ic antiarrhythmic agent) administration (35). The EpA960 model (currently unavailable due to extinguished transgene expression) based on Cre-mediated recombination to express 960 interrupted CUG repeats displayed severe cardiac conduction phenotypes and alternative splicing changes in the heart (36). The DMSXL model expressing more than 1000 CUG repeats from a BAC containing an expanded human DMPK gene showed weak splicing changes and cardiac conduction abnormalities in aged mice in response to flecainide administration (37).

In this study, we developed a heart-specific mouse model of DM1 based on tetracycline-inducible expression of RNA containing 960 interrupted CUG repeats in the context of human DMPK exons 11-15. Expression of pathogenic CUGexp RNA reproduces many of the cardiac conduction defects and propensity for atrial arrhythmias as observed in individuals affected by DM1. The mice also show MBNL1 and MBNL2 protein colocalization with CUGexp RNA foci and robust splicing defects characteristic of DM1 cardiac tissue. Importantly, the observed electrophysiological, cellular, and molecular phenotypes are reversible upon turning off expression of CUGexp RNA. By RNA-Seq analysis we identified several gene expression and alternative splicing changes in genes associated with the regulation of ion transport in atria and ventricles, which could be associated with phenotypic disease-relevant manifestations. Isolated atrial cardiomyocytes from repeat expressing mice showed calcium-handling defects suggesting a potential mechanism contributing to atrial arrhythmogenesis.

\section{Results}

Inducible and reversible expression of CUGexp RNA in atrial and ventricular cardiomyocytes. We developed a mouse model that uses a bitransgenic system for tetracycline-inducible and cardiomyocyte-specific expression of CUGexp RNA. First, we generated transgenic mouse lines bearing the TREDT960I transgene containing 960 interrupted CTG repeats in the context of human DMPK exons 11-15 (38) (Figure 1A). The expression of the transgene is regulated by a minimal CMV promoter fused to a tetracycline response element (TRE). To make the expression of CUGexp RNA heart-specific, TREDT960I mice were crossed with a MHCrtTA line, in which the expression of a modified reverse tetracycline transactivator is regulated by an $\alpha$ myosin heavy chain ( $\alpha \mathrm{MHC}$ ) promoter (39).

Bitransgenic animals used in this study, referred to as CUG960, are homozygous for the TREDT960I transgene and hemizygous for the MHCrtTA transgene. CUG960 animals were given doxycycline-containing (dox-containing) chow to induce expression of CUGexp RNA containing 960 CUG repeats. Mice hemizygous for the MHCrtTA transgene and given dox chow were used as controls for the effects of dox and rtTA expression in cardiomyocytes. CUG960 and MHCrtTA mice were given $2 \mathrm{~g} / \mathrm{kg}$ dox chow starting on postnatal day 1 (PN1) for a period of 2 months, after which mice were characterized for DM1-associated cardiac phenotypes (Figure 1B). To evaluate reversibility of disease features in response to diminished CUGexp RNA expression, animals were switched to standard chow for either 1 or 2 months and analyzed using the same battery of characterization studies.

Relative expression levels of CUGexp RNA were evaluated by quantitative reverse-transcription PCR (RT-qPCR) analysis in the atria and ventricles of the heart. In comparison with controls, CUG960 + dox mice exhibited a robust induction of transgene expression, which was extinguished in response to dox withdrawal (Figure 1C). 
A
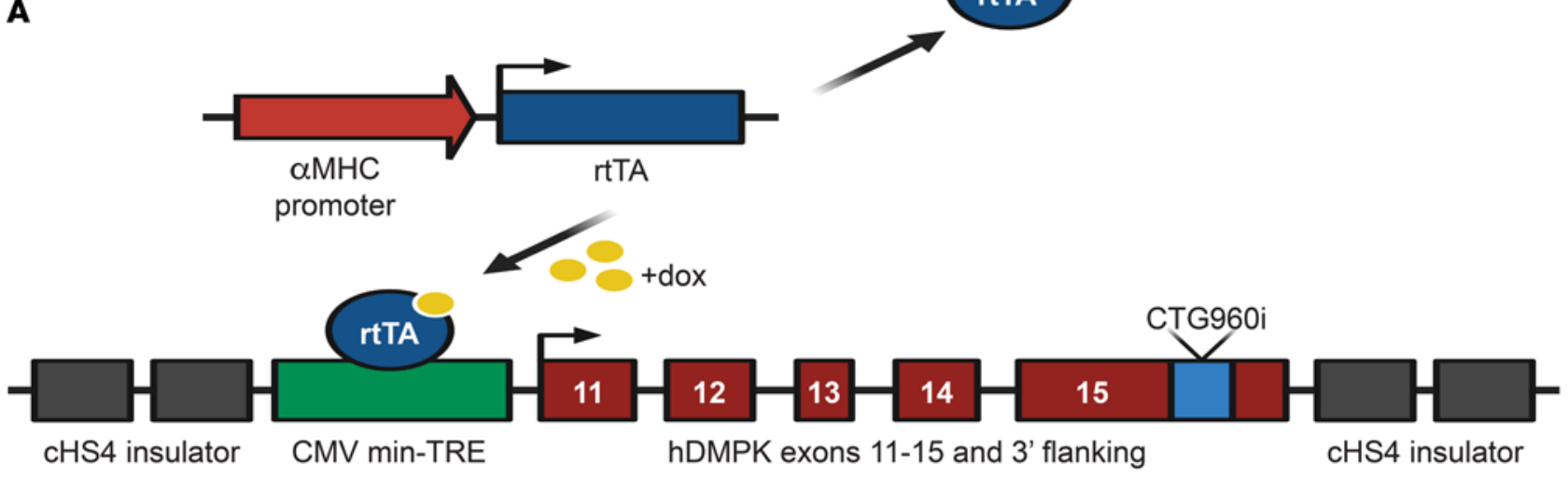

B

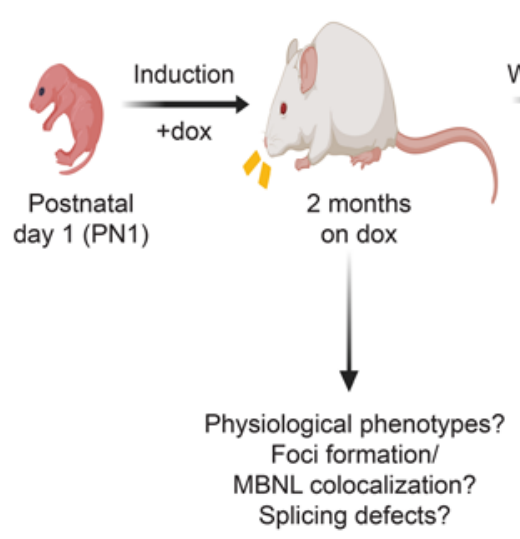

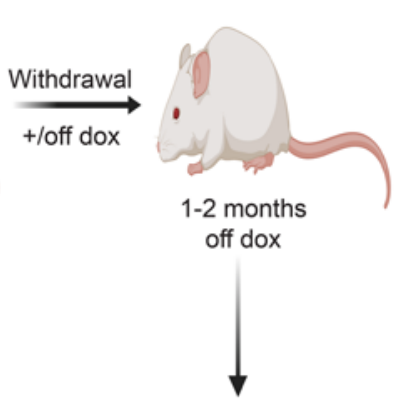

Physiological phenotypes? Foci formation/ MBNL colocalization? Splicing defects?
C

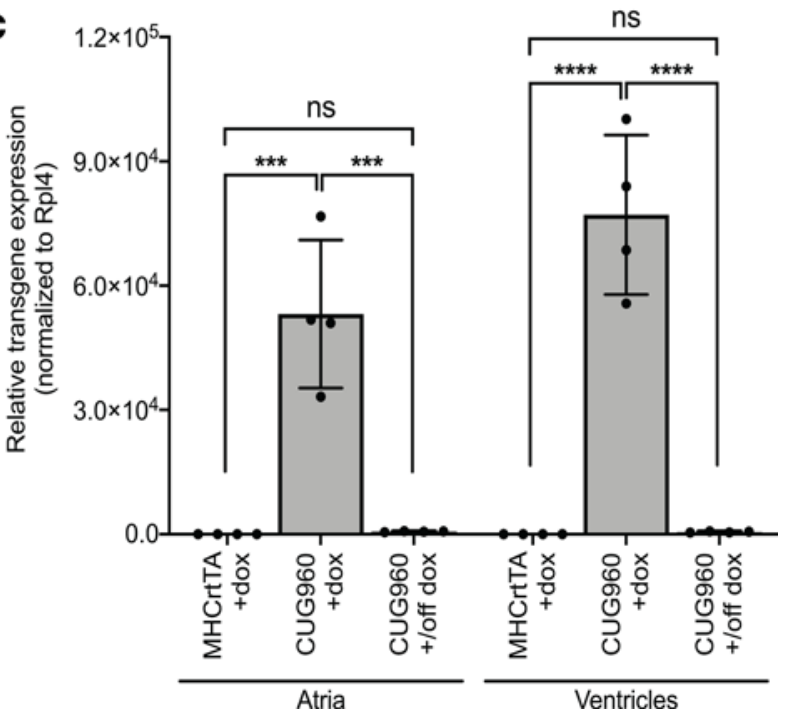

Figure 1. Bitransgenic mouse model for inducible and heart-specific expression of CUGexp RNA. (A) The TREDT960l transgene consists of a minimal CMV promoter fused to a tetracycline response element regulating doxycycline induction of RNA containing 960 interrupted CUG repeats in the context of human DMPK exons 11-15. The expression of reverse tetracycline transactivator (rtTA) transgene is driven by a cardiomyocyte-specific $\alpha$ myosin heavy chain promoter. (B) Animals were given $2 \mathrm{~g} / \mathrm{kg}$ dox food for induction of CUG repeat RNA expression beginning at PN1 and characterized for DM1-associated cardiac manifestations. Animals were switched to standard chow to evaluate reversal of disease features in response to cessation of CUCexp RNA expression. (C) RT-qPCR analysis of transgene mRNA expression in atria and ventricles of CUG960 mice in response to dox induction since PN1 for 2 months and withdrawal for 2 months in comparison with MHCrtTA +dox control mice. $m R p / 4$ was used as an internal control for normalization. $n=4$ animals per group. Data represent the mean \pm SD and were analyzed using 1-way ANOVA followed by Tukey's test for multiple comparisons. ${ }^{* * *} P<0.001,{ }^{* * * *} P<0.0001$. CUGexp, expanded CUG repeat; DM1, myotonic dystrophy type 1; dox, doxycycline; PN1, postnatal day 1.

CUG960 mice displayed physiological phenotypes associated with DM1 cardiac disease. We evaluated CUG960 + dox mice for the cardiac disease features observed in DM1. Cardiac contractility and morphology were measured by M-mode echocardiography. CUG960 + dox mice displayed no significant differences in ejection fraction, fractional shortening, or intraventricular diameter (Supplemental Figure 1, A-D; supplemental material available online with this article; https://doi.org/10.1172/jci.insight.143465DS1). In comparison with MHCrtTA +dox controls, CUG960 +dox mice exhibited significantly increased left ventricular posterior wall diameter (Supplemental Figure 1, E and F) and larger hearts (approximately 28\%) with significantly increased heart weight/tibia length ratios (Supplemental Figure 1G), in agreement with the phenotypes observed previously in a compound muscleblind-knockout model (31).

We examined CUG960 + dox mice for changes in cardiac conduction intervals by surface ECG recordings in anesthetized mice. In comparison with MHCrtTA + dox controls, CUG960 + dox mice did not show prolongation of the RR (Figure 2A) or PR (Figure 2B) intervals, as observed in DM1. However, all CUG960 + dox mice showed robust ventricular conduction defects with prolongation of the QRS (approximately $27 \%$ 
A

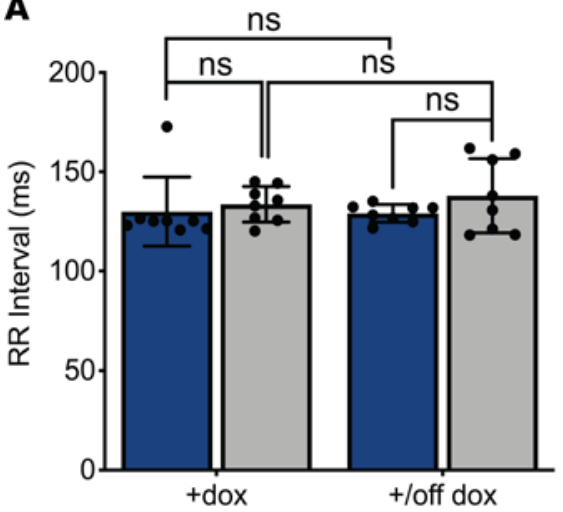

C

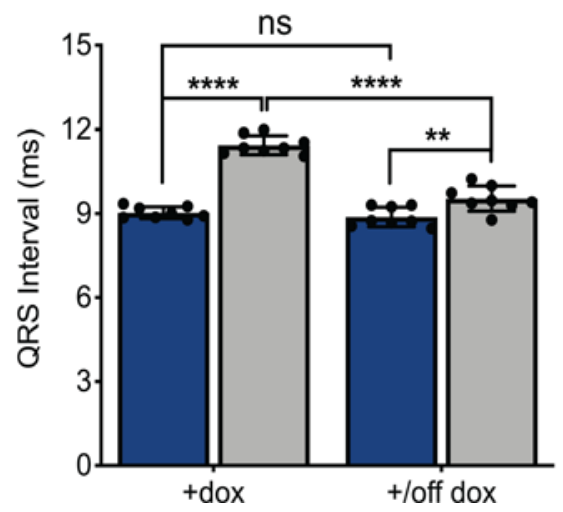

B
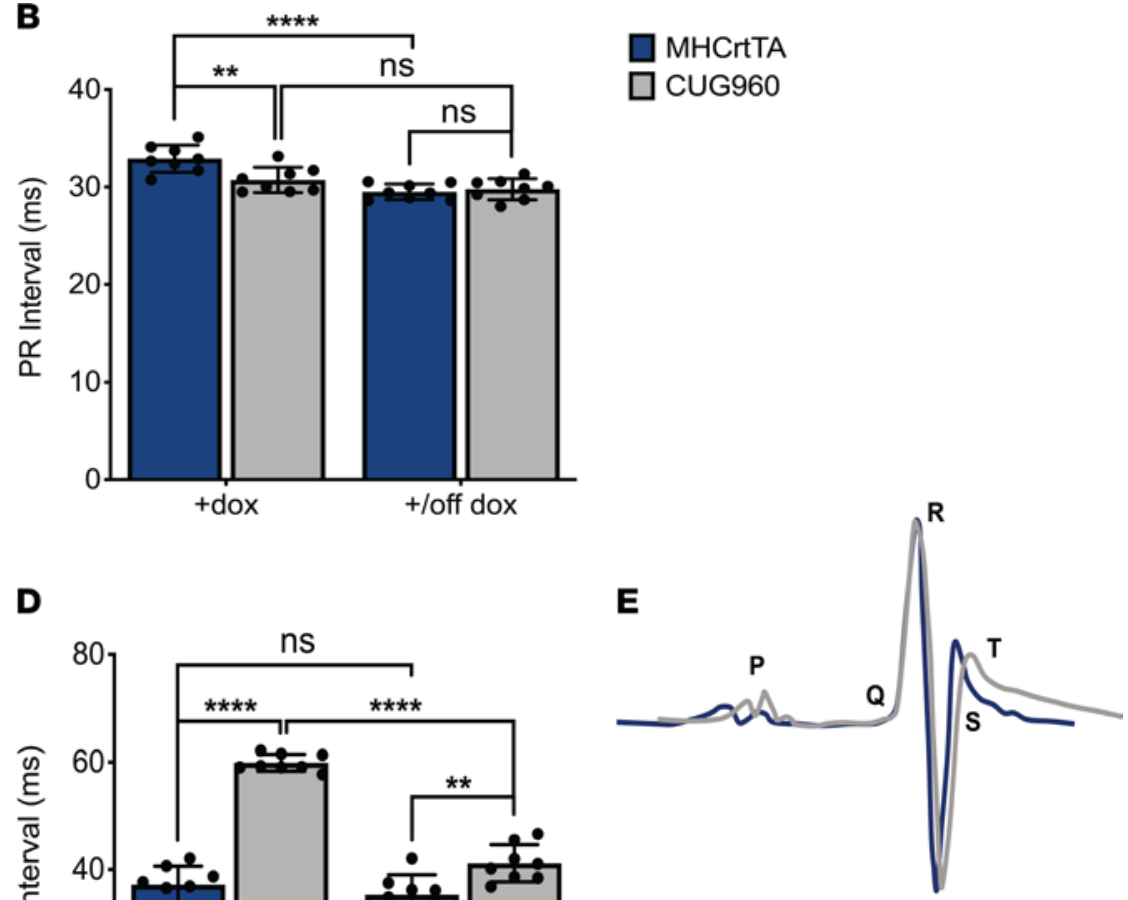

D

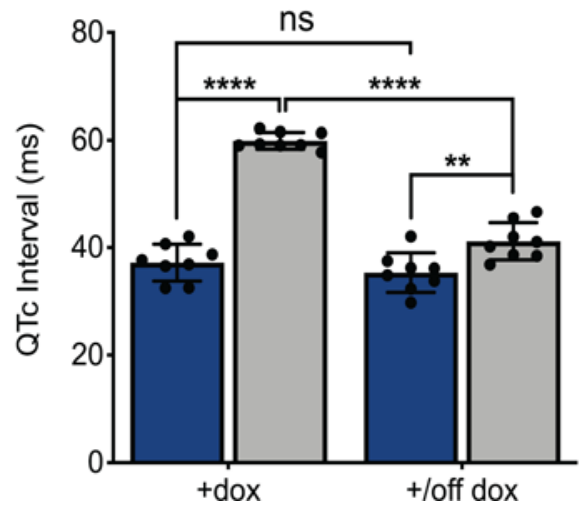

MHCrtTA

CUG960

Figure 2. CUG960 mice display reversible conduction abnormalities associated with DM1. Surface ECC recordings were obtained to evaluate conduction intervals in CUC960 and MHCrtTA control mice given dox chow starting at PN1 for 2 months and then switched to regular chow for 2 months: (A) RR interval (B) PR interval (C) QRS interval and (D) corrected QT interval (QTC). $n=8$ animals per group. (E) Overlap of representative ECC tracings from MHCrtTA +dox and CUC960 +dox mice. Data represent the mean \pm SD and were analyzed using 2-way ANOVA followed by Tukey's test for multiple comparisons. ${ }^{*} P<0.01$, ${ }^{* * * *} P<0.0001$. DM1, myotonic dystrophy type 1; PN1, postnatal day 1; dox, doxycycline.

increase) (Figure 2, C and E) and QTc (approximately 61\% increase) (Figure 2, D and E) intervals in comparison with control animals. Interestingly, CUG960 mice taken off dox chow for 2 months showed rescue of these parameters in response to diminished CUGexp RNA expression (Figure 2, C and D).

We evaluated whether conduction defects are also observed in animals induced to express CUGexp RNA as adults to exclude any effects of transgene expression on postnatal heart development. CUG960 and MHCrtTA control mice were analyzed by ECG before CUGexp RNA induction at 2 months of age, then after induction on $2 \mathrm{~g} / \mathrm{kg}$ dox chow for 2 months and then again after a switch to regular chow for 2 months to evaluate reversibility of any observed phenotypes (Supplemental Figure 2A). We observed similar conduction defects - prolongation of QRS and QTc intervals, only on induction of transgene expression, which were rescued following dox withdrawal (Supplemental Figure 2, B and C).

Across multiple cohorts, a proportion of CUG960 + dox mice induced at PN1 (approximately 15\%), but none of the MHCrtTA + dox control mice, displayed supraventricular arrhythmias during 1.5-2 minutes of surface ECG recordings (Figure 3A). To evaluate the predisposition of CUG960 +dox mice to atrial arrhythmias in comparison with MHCrtTA +dox controls, we performed intracardiac pacing on 2-monthold CUG960 and MHCrtTA mice, given dox chow beginning at PN1. The incidence of reproducible pacing induced atrial arrhythmias was significantly higher in CUG960 +dox mice $(75 \%, n=12)$ than in MHCrtTA + dox controls $(22.2 \%, n=9)$ (Figure 3, B and C). To evaluate reversibility of this phenotype, CUG960 and MHCrtTA animals were taken off dox chow for 1 month after dox induction. In response to diminished CUGexp RNA expression, we observed no significant differences between CUG960 and MHCrtTA animals (Figure 3, B and D), indicating rescue of the increased predisposition to atrial arrhythmias in CUG960 mice.

CUG960 mice exhibited RNA foci and DM1-associated splicing defects. Aggregation of pathogenic CUGexp RNA into nuclear foci and sequestration of MBNL proteins are key disease hallmarks of DM1, observed 
A

MHCrtTA +dox
control
(Sinus Rhythm)

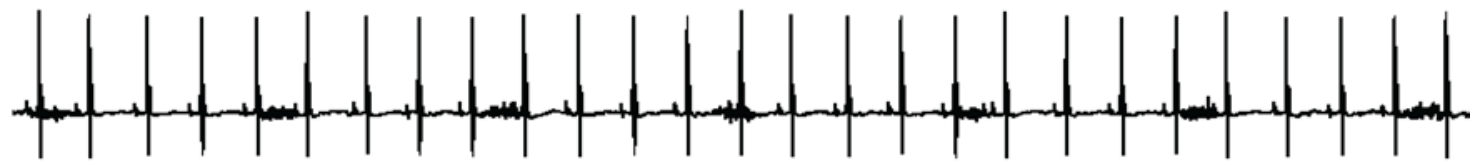
CUG960 +dox \#1 (Supraventricular Tachycardia)

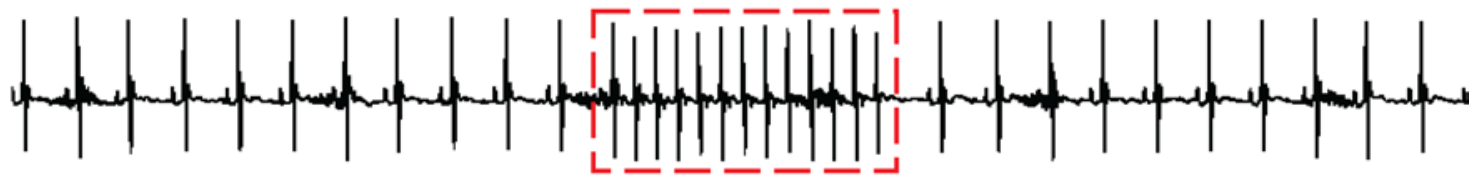
CUG960 +dox \#2 (Premature Atrial Contraction)

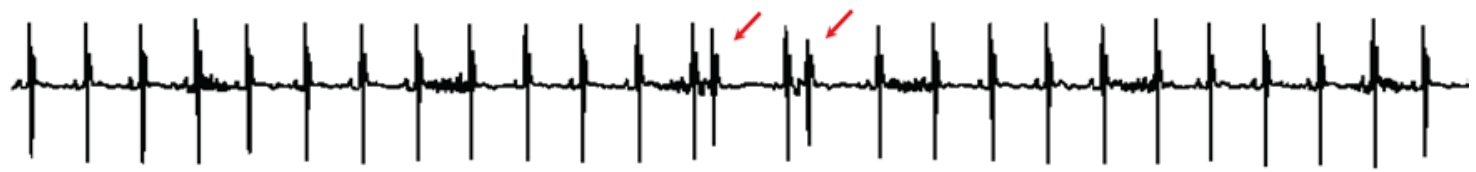

B

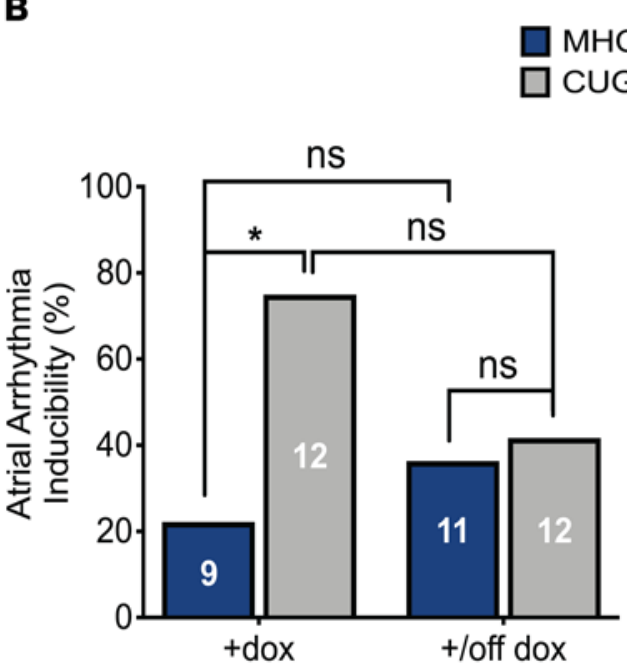

C
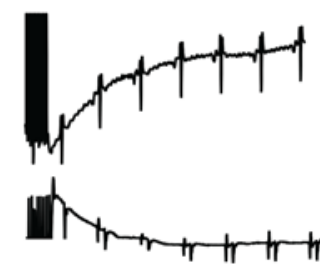

D

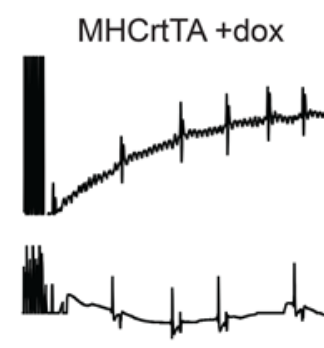

MHCrtTA +/off dox
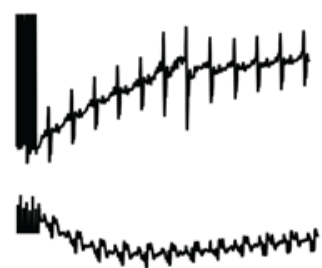

Atrial

Electrogram

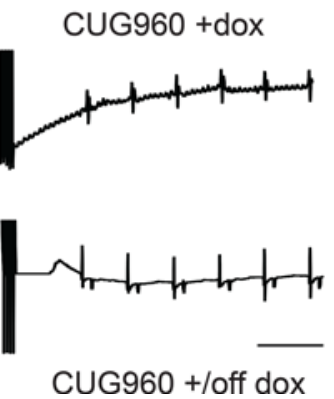

Surface ECG

Surface ECG

Atrial Electrogram

Figure 3. CUC960 mice exhibit an increased and reversible predisposition to atrial arrhythmias. (A) Baseline arrhythmias observed in surface ECC recordings for CUC960 +dox mice in comparison with MHCrtTA +dox controls. (B) Incidence of atrial arrhythmias induced by electrical pacing in CUC960 vs. MHCrtTA control mice in response to dox induction initiated at PN1 for 2 months and dox withdrawal for 1 month after 2 months of induction initiated at PN1. (C) Representative recordings of surface and intracardiac atrial electrograms in CUC960 +dox and MHCrtTA +dox control mice after rapid atrial pacing. (D) Representative recordings of surface and intracardiac atrial electrograms in CUC960 +/off dox and MHCrtTA +/off dox control mice after rapid atrial pacing. Numbers of animals analyzed are indicated in corresponding bars. Data were analyzed using Fisher's exact test. ${ }^{*} P<0.05$. Scale bar: 200 milliseconds. PN1, postnatal day 1; dox, doxycycline.

in DM1 cardiac tissues (6). To determine whether CUG960 mice display nuclear RNA foci and MBNL colocalization, we performed FISH using Tye-563-labeled (CAG) 5 locked nucleic acid probes targeting CUGexp RNA combined with IF for MBNL1 or MBNL2. Hearts from CUG960 +dox mice induced to express CUGexp RNA displayed multiple distinct RNA foci in nuclei with colocalization of MBNL1 and MBNL2 proteins (Figure 4, D-F, and Supplemental Figure 3, D-F) and no foci were found to be present in hearts of MHCrtTA +dox control mice (Figure 4, A-C, and Supplemental Figure 3, A-C). CUG960 mice induced with dox chow for 2 months and then switched to regular chow for 2 months (+/off dox) showed no foci formation or MBNL aggregation (Figure 4, G-I, and Supplemental Figure 3, G-I), thereby demonstrating the reversibility of these phenotypes in response to diminished CUGexp RNA expression. Previous studies have implicated elevated CELF1 protein levels in DM1 hearts with disease pathology $(10,40)$. We evaluated CELF1 protein levels in atria and ventricles of CUG960 + dox and MHCrtTA +dox control mice. In comparison with controls, CUG960 + dox mice did not show a statistically significant change in CELF1 protein levels but variability in the results suggests that some induced animals showed higher levels of CELF1 protein in atria compared with control mice (Supplemental Figure 4, A and B).

A downstream consequence of CUGexp RNA expression is disruption of developmentally regulated alternative splicing with target transcripts showing reversion to fetal isoforms. We evaluated CUG960 +dox 

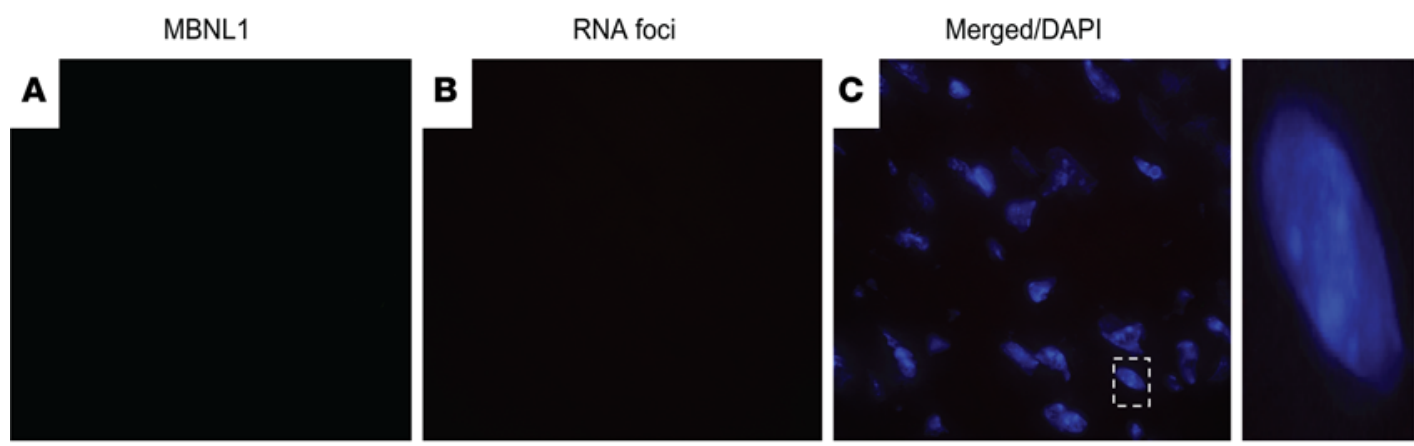

MHCrtTA
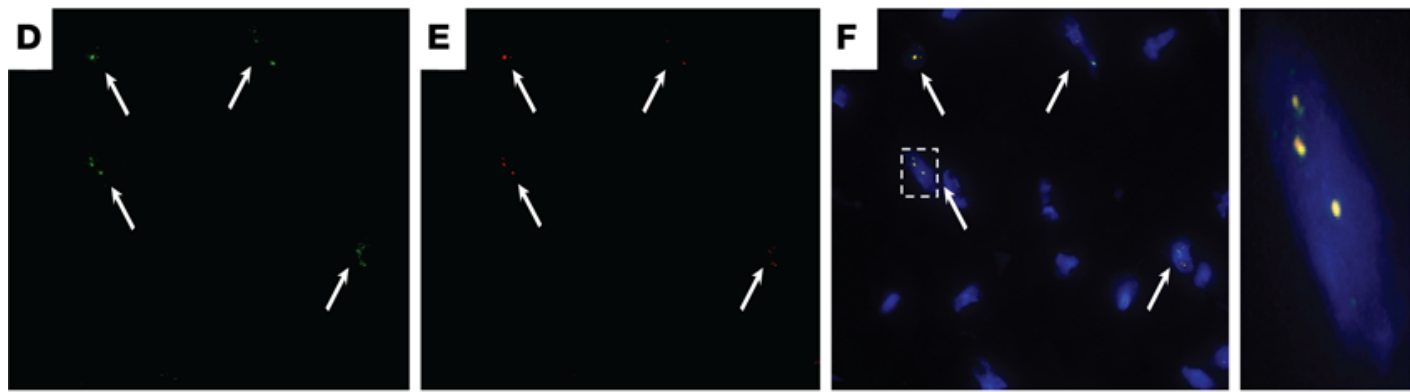

+ dox
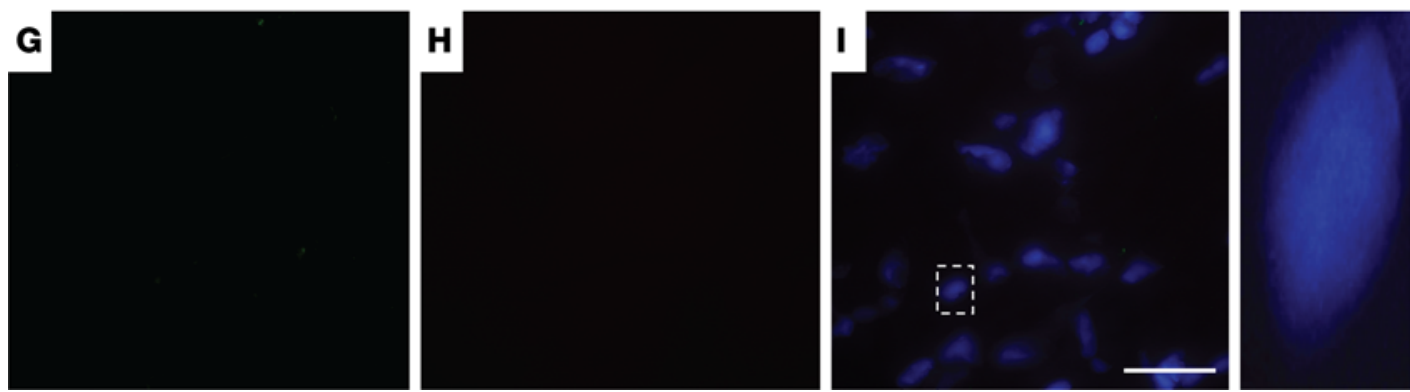

CUG960

+ dox

CUG960

+/off dox

Figure 4. CUG960 mice display reversible nuclear RNA foci formation and MBNL1 colocalization. RNA FISH with a probe targeting CUG repeat RNA combined with immunofluorescence for MBNL1 in the ventricles of CUG960 mice in response to dox induction (D-F) and withdrawal (G-I) in comparison with MHCrtTA + dox control mice (A-C). Arrows indicate RNA foci colocalized with MBNL1 staining. Scale bar: 25 microns. dox, doxycycline.

mice for splicing events that are (a) conserved in mouse postnatal cardiac development (41) and (b) known to be affected in DM1 individuals (42) and Mbnl-knockout mouse models (31, 43). Four splicing events were selected based on these criteria: Sorbs1 exon 25, Spag9 exon 31, Tnt2 exons 4 and 5, and Mef2d $\beta$-exon. In comparison with MHCrtTA + dox controls, CUG960 + dox mice showed strong disruption of alternative splicing events in both the atria and ventricles, shifting toward fetal splicing patterns, and these splicing defects could be completely rescued upon turning off transgene expression (Figure 5). To determine the dynamics of the induction and reversibility of splicing defects in response to CUGexp RNA expression, we performed a time course experiment in which adult 6-month-old CUG960 mice were fed dox chow for 3 days, 1 week, or 4 weeks and taken off dox chow for 5 days, 10 days, 16 days, or 4 weeks after 4 weeks of induction (Supplemental Figure 5A). We isolated ventricular RNA from the mice at these time points and evaluated them for the Sorbs 1 exon 25 and Spag9 exon 31 splicing events. Interestingly, CUG960 +dox mice exhibited a complete splicing switch to the DM1 pattern as early as 3 days on dox chow and nearly complete rescue of these defects by 5 days after dox withdrawal (Supplemental Figure 5B).

CUGexp RNA expression in the heart led to transcriptomic alterations affecting ion transport genes. To broadly identify transcriptome changes potentially contributing to the phenotypic manifestations in CUGexp RNA-expressing mice, we performed RNA-Seq analysis on poly(A)-selected RNA from atria and ventricles of 3 CUG960 +dox and 3 MHCrtTA +dox control mice after 10 weeks of induction starting on PN1 (Supplemental Table 1). We obtained an average of 130 million paired-end 150 base pair (bp) reads with $75 \%$ or greater reads uniquely mapping to the mouse genome. The quality and depth of the sequencing data were sufficient to identify gene expression and alternative splicing changes associated with CUGexp RNA expression in the heart. For both gene expression (Supplemental Figure 6, A and B) and alternative 

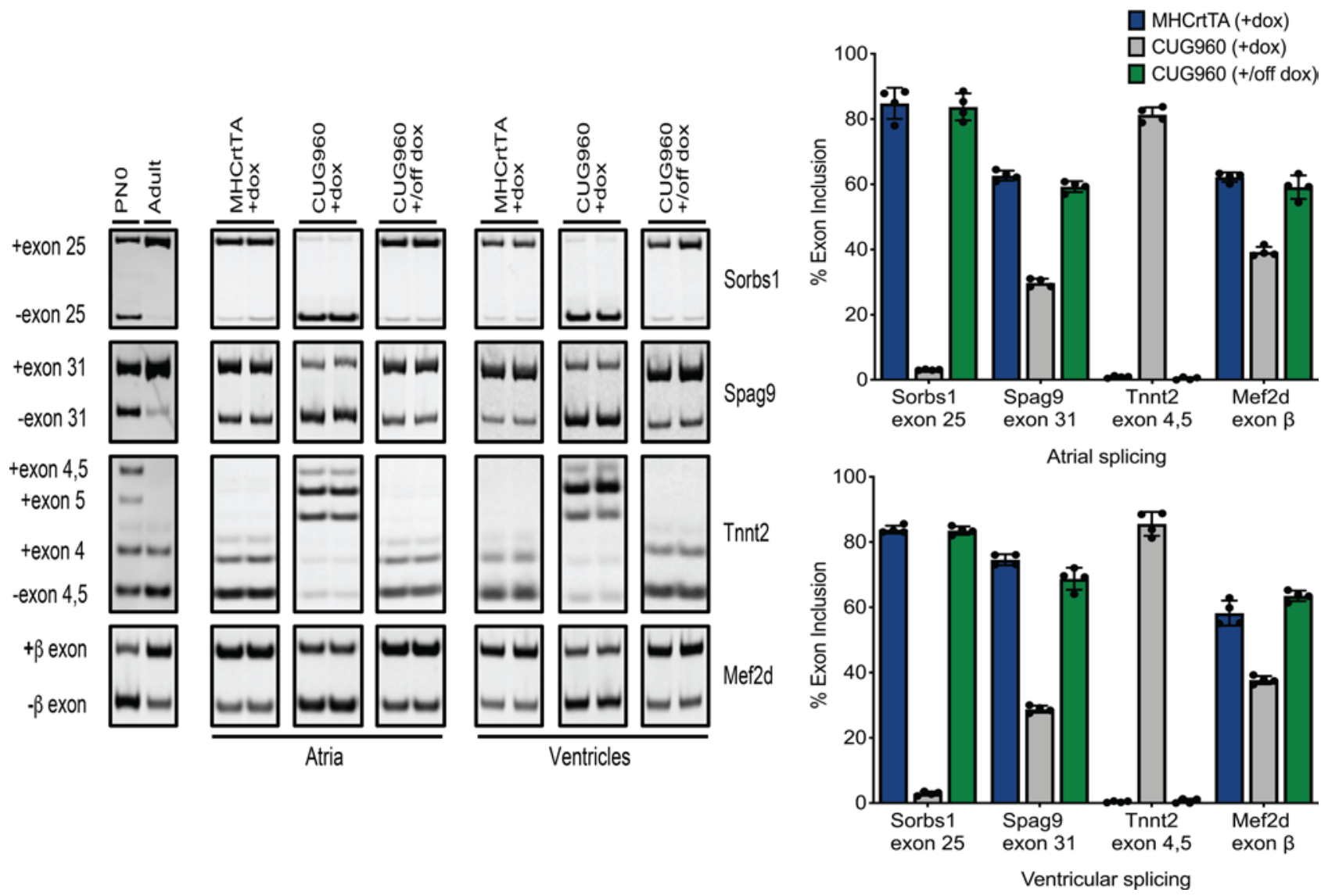

Figure 5. CUC960 mice exhibit strong and reversible DM1-associated splicing defects. Representative RT-PCRs with quantification showing strong reversion to fetal splicing patterns for Sorbs1 exon 25, Spag9 exon 31, Tnnt2 exons 4 and 5, and the Mef2d $\beta$-exon in atria and ventricles of CUC960 +dox mice in comparison with MHCrtTA +dox controls. Splicing defects are completely rescued in CUC960 mice after dox withdrawal. $n=4$ animals per group. Data represent the mean \pm SD. DM1, myotonic dystrophy type 1; PNO, postnatal day 0 heart; Ad, adult heart ventricle; dox, doxycycline.

splicing (Supplemental Figure 6, C and D) data sets, we observed strong correlation between biological replicates indicating high levels of reproducibility.

We identified 253 atrial (169 upregulated and 84 downregulated) and 248 ventricular (167 upregulated and 81 downregulated) differentially expressed genes (adjusted $P$ value $<0.05$, fold change $\geq 1.5$ ) between CUG960 +dox and MHCrtTA +dox control mice (Figure 6A). While genes showing differential expression induced by CUGexp RNA in the atria and ventricles showed less than 30\% overlap (Figure 6B), gene ontology analysis revealed enrichment for similar functions of "muscle contraction," "ion transport," and "regulation of membrane potential" (Figure 6, C and D). We focused on the category of ion transport genes given their role in regulation of the cardiac action potential and previous associations with cardiac conduction disease (see Discussion). Candidate genes of interest (Hcn4, Gja5, Scn10a, and Junctin) with distinct expression patterns in atria or ventricles in CUG960 + dox mice were validated by RT-qPCR analysis (Figure 6E).

In comparison with MHCrtTA +dox controls, we identified 893 events in atria and 849 events in ventricles of CUG960 + dox mice that show differential alternative splicing patterns (FDR $<0.05, \Delta \mathrm{PSI} \geq 15 \%$ ) (Figure 7A). Given the large number of cassette exons affected relative to the other types of alternative splicing events (approximately 75\%), we focused on this category for further analysis. In contrast to differential gene expression, we found that genes affected at the level of alternative splicing in atria and ventricles showed primarily similar profiles with approximately $60 \%$ overlap (Figure 7B). There was minimal overlap observed between the genes affected at the level of mRNA abundance and alternative splicing in both atria and ventricles (Supplemental Figure 6, E and F), indicating that these groups of genes are affected at distinct nodes of regulation. A total of 3 genes - Necap2 (atria), Coq8a (atria and ventricles), and Pdlim7 (atria and ventricles) - exhibited alternative splicing changes associated with the introduction of a premature stop codon leading to changes in expression levels due to nonsense mediated decay. Gene ontology 
A
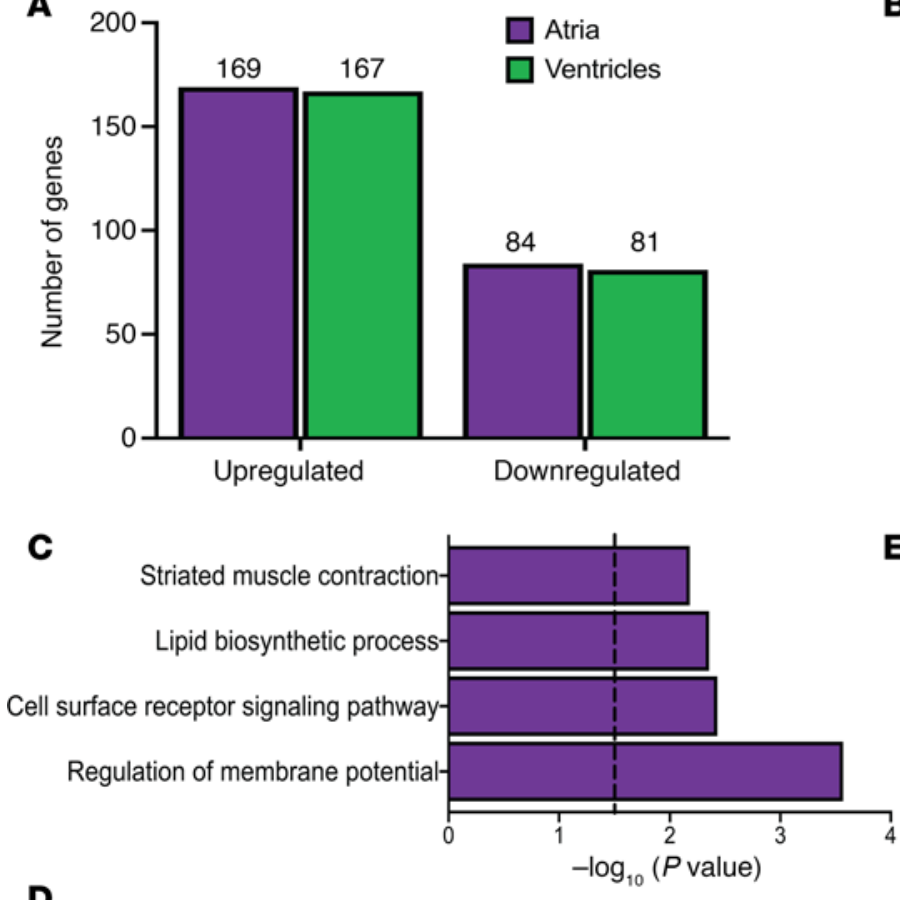

D

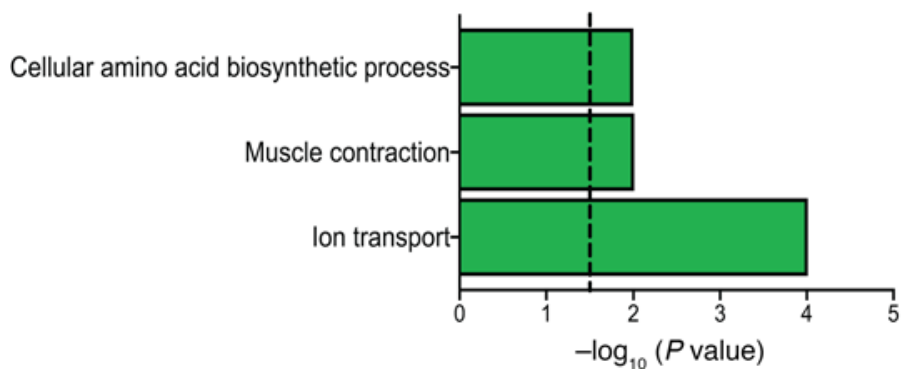

B
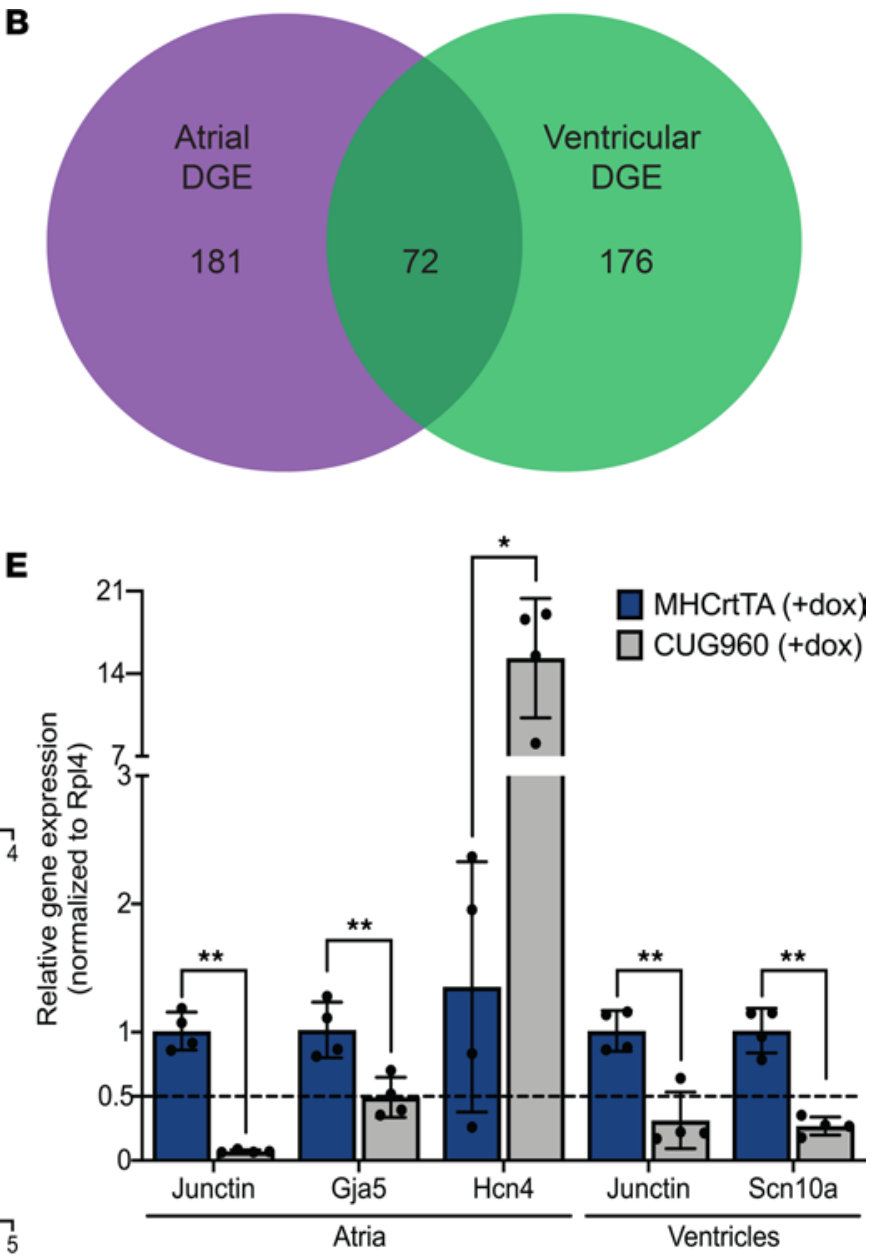

Figure 6. CUGexp RNA induced differential gene expression in atria and ventricles. (A) Number of upregulated/downregulated genes in atria and ventricles of CUG960 +dox mice. (B) Overlap observed between differentially expressed genes in atria and ventricles of CUC960 +dox mice. Cenes showing differential gene expression changes in (C) atria and (D) ventricles in CUG960 +dox mice were evaluated for enrichment of gene ontology functional terms using DAVID platform. Cutoff $>-\log (P$ value $)=1.5$. (E) RT-qPCR-based validation of candidate ion transport genes, Hcn4, Gja5, Scn10a, and Junctin, showing gene expression changes in atria and ventricles of CUG960 +dox mice in comparison with MHCrtTA +dox controls. mRp/4 was used as an internal control for normalization. $n=4$ animals per group. Data represent the mean $\pm S D$ and were analyzed using 2-tailed $t$ test. ${ }^{*} P<0.05,{ }^{* *} P<0.01$. CUGexp, expanded CUG repeat; dox, doxycycline.

analysis of genes undergoing changes in alternative splicing in atria and ventricles showed enrichment for common functional terms associated with "cytoskeleton organization," "vesicle mediated transport," "positive regulation of ion transport," and "heart development" (Figure 7, C and D). Similar to our differential gene expression analysis, we focused on alternative splicing events affecting genes involved in regulation of cardiac ion transport and previously implicated in conduction disorders - Scn5a, Kcnd3, Kcnip2, Ryr2, and Camk2d (Figure 7E). Alternative splicing defects in these genes involved in the regulation of cardiac action potential (Scn5a, Kcnd3, and Kcnip2) and calcium handling (Ryr2 and Camk2d) lead to production of isoforms with differential properties which could play a pathogenic role in adult tissue (see Discussion).

Isolated atrial cardiomyocytes from CUG960 mice showed calcium-handling defects consistent with CUGexp $R N A$-induced transcriptomic alterations. Defects in calcium handling are significant contributors to the pathophysiology of atrial arrhythmias (44). Given the increased predisposition to atrial arrhythmias and expression or alternative splicing differences in calcium-handling genes observed in CUGexp RNA-expressing mice, we performed calcium imaging studies in atrial cardiomyocytes isolated from CUG960 +dox mice in comparison with MHCrtTA + dox controls to probe for perturbations in calcium cycling. In comparison with controls, CUG960 + dox mice did not show differences in spontaneous calcium spark frequency or sarcoplasmic reticulum (SR) load (Supplemental Figure 7, A-C). However, CUG960 +dox mice exhibited a higher incidence of diastolic spontaneous calcium waves (Figure 8, A and B) and increased systolic 
A
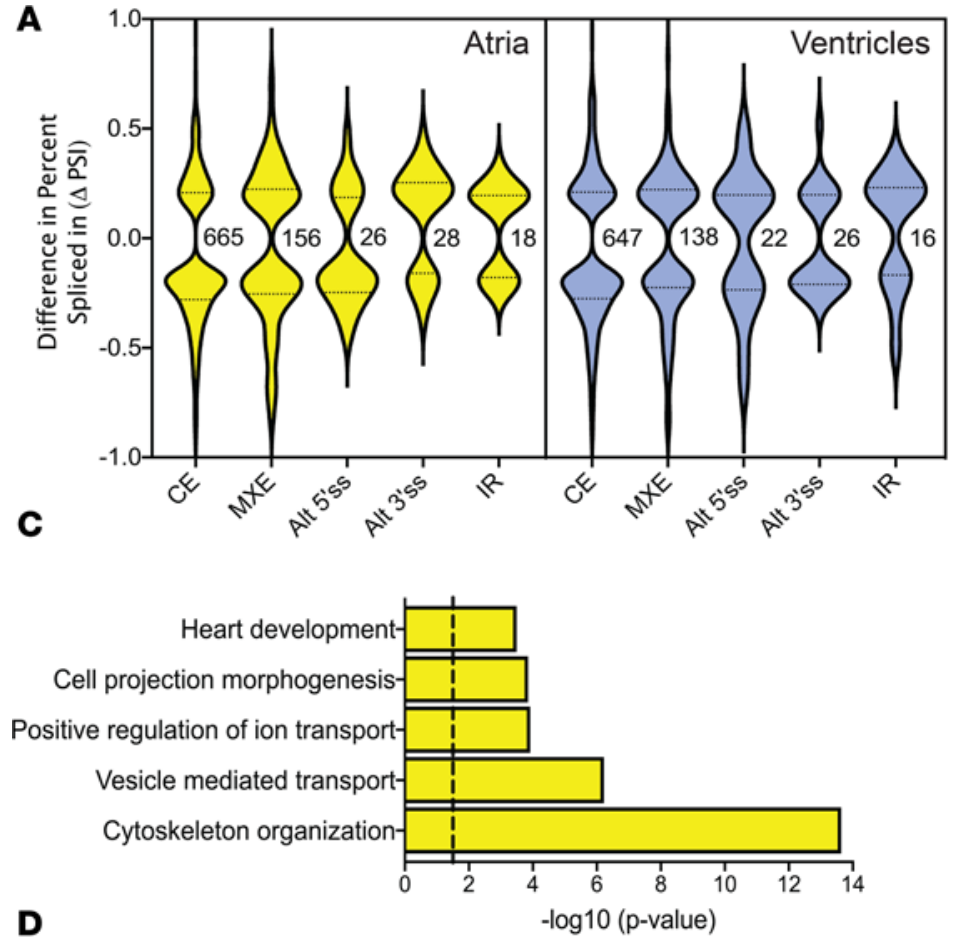

B

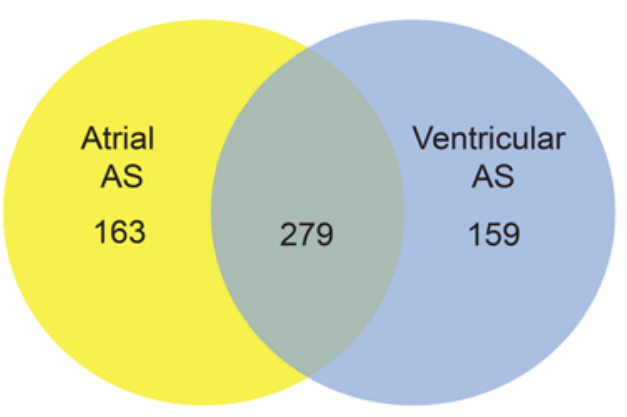

$\mathbf{E}$
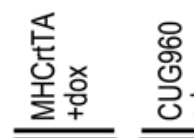

exon $6 A$

exon $6 \mathrm{~B}$
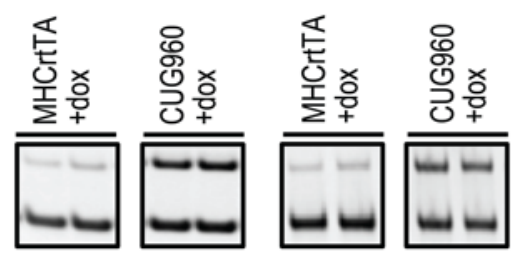

Scn5a

texon 6
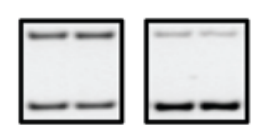

texon 3

-exon 3
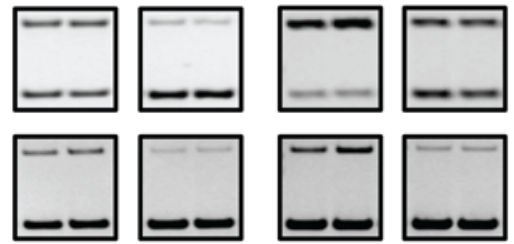

Kcnd3

+exon 4,5

+exon 5

-exon 4,5
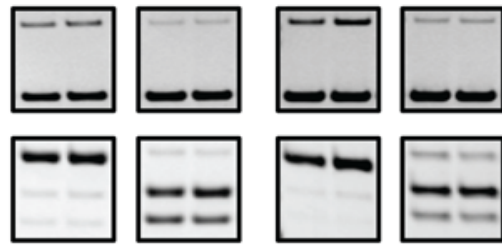

Kcnip2
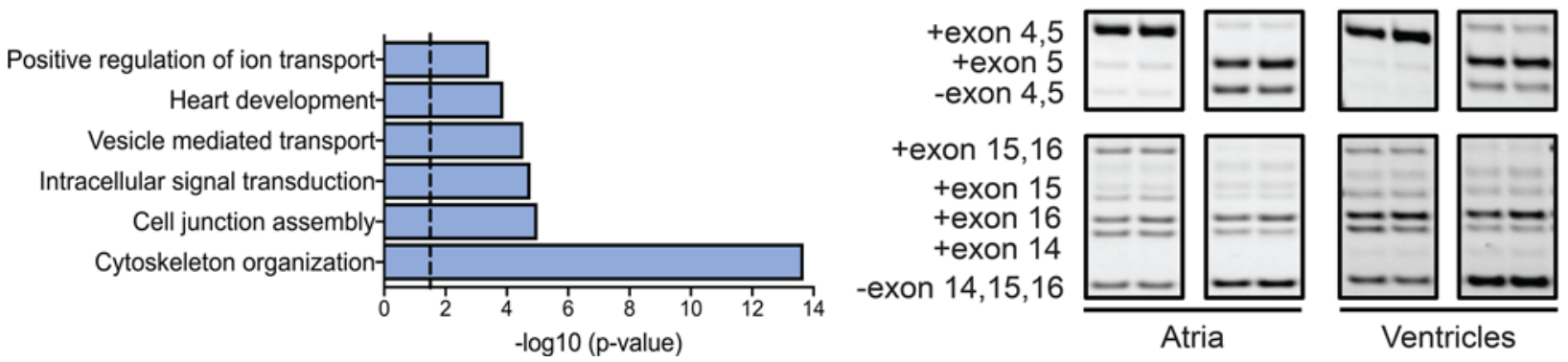

Ryr2

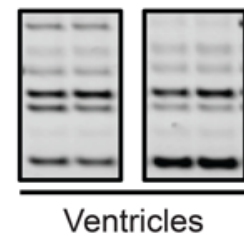

Camk2d

Figure 7. CUGexp RNA induced alternative splicing changes in atria and ventricles. (A) Violin plots depicting the distribution of splicing events based on $\triangle \mathrm{PSI}$ and types of AS changes observed in atria and ventricles of CUC960 + dox mice. (B) Overlap observed between genes showing alternative splicing changes in atria and ventricles of CUG960 +dox mice. Genes showing differential alternative splicing changes in (C) atria and (D) ventricles in CUC960 +dox mice were evaluated for enrichment of gene ontology functional terms using DAVID platform. Cutoff $>-\log (P$ value $)=1.5$. (E) Representative RT-PCRs showing alternative splicing changes for candidate ion transport genes, Scn5a, Kcnd3, Kcnip2, Ryr2, and Camk2d, in atria and ventricles of CUG960 +dox mice in comparison with MHCrtTA +dox controls. $n=2$ animals per group. CUGexp, expanded CUG repeat; dox, doxycycline; CE, cassette exons; MXE, mutually exclusive exons; Alt 5'ss/Alt 3'ss, alternative 5'/alternative 3' splice site; IR, intron retention.

calcium transient amplitude (Figure 8C), suggestive of increased RyR2 activity. Additionally, myocytes demonstrated increased $\mathrm{Na}^{+} / \mathrm{Ca}^{2+}$ exchanger (NCX) (Figure 8, D and E) and decreased sarcoendoplasmic reticulum calcium ATPase (SERCA) current (Figure 8F). In response to diminished CUGexp RNA expression, CUG960 mice displayed rescue of increased calcium waves incidence (Figure 8, A and B) and decreased SERCA current (Figure 8F).

\section{Discussion}

Despite $50 \%$ of patients with DM1 having cardiac manifestations, the tissue-specific mechanisms that contribute to cardiac disease features are not well understood. To model DM1-associated RNA toxicity in the heart, we developed transgenic mice for inducible and cardiomyocyte-specific expression of 960 CUG repeats in the natural context within human DMPK exon 15. In comparison with controls, CUG960 +dox mice exhibited DM1-associated prolongation of QRS and QTc conduction intervals. CUG960 +dox mice showed spontaneous supraventricular arrhythmias and a higher predisposition to pacing-induced atrial arrhythmias. CUG960 +dox mice also showed the presence of nuclear foci, colocalization of MBNL1 and MBNL2 proteins with the foci, and strong reversion to fetal alternative splicing patterns in both the atria 
A MHCrtTA +dox
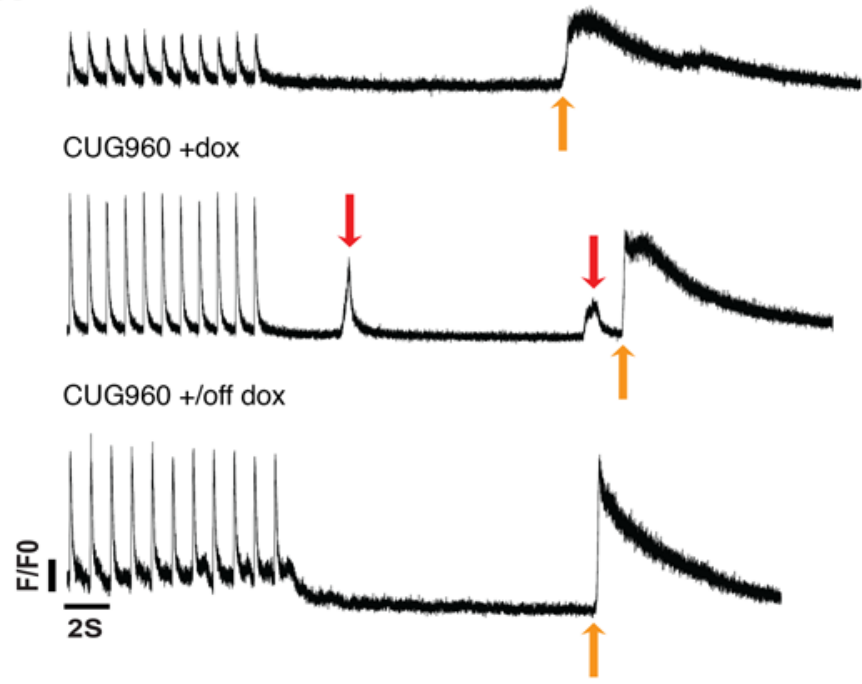

C

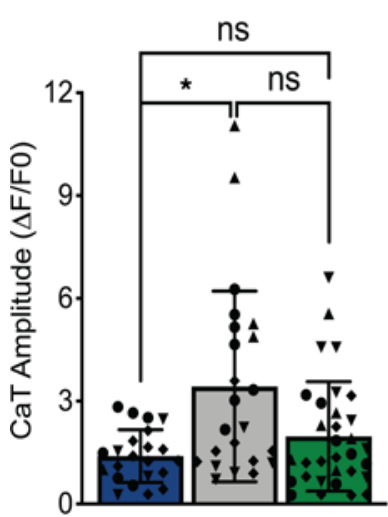

D
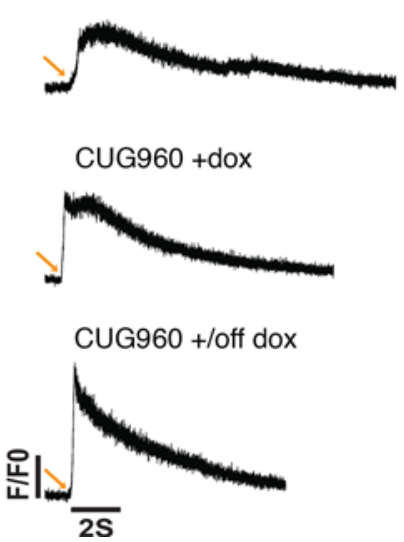

B

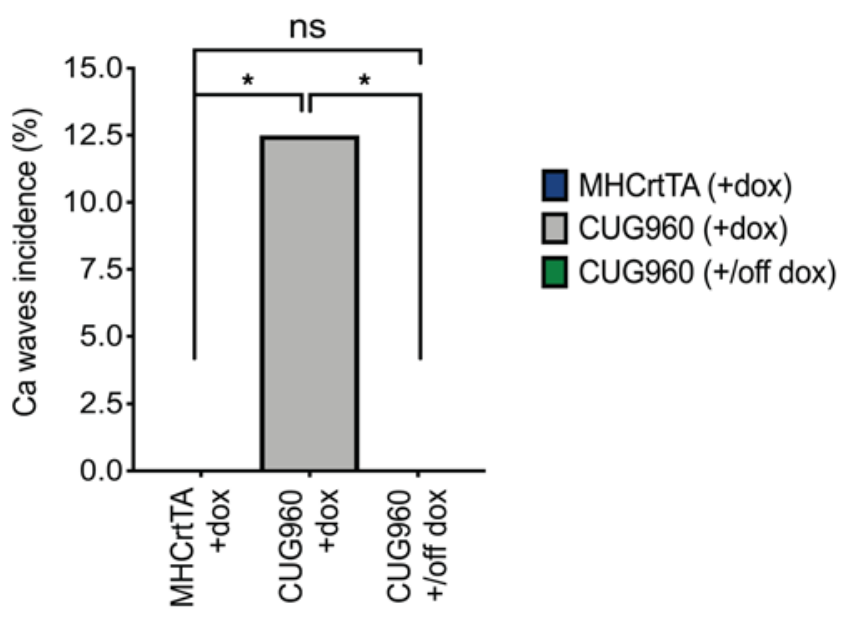

E

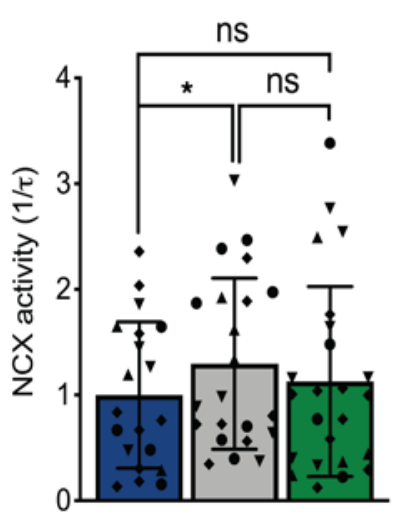

$\mathbf{F}$

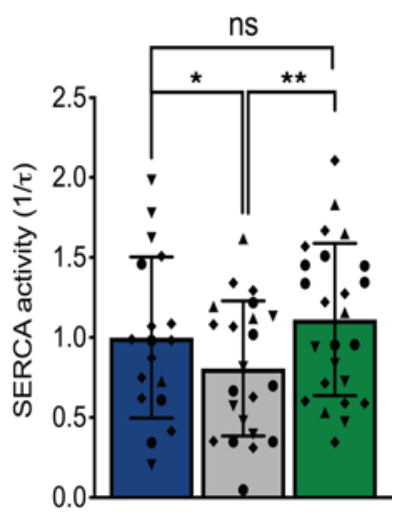

Figure 8. $\mathrm{Ca}^{2+}$ handling is altered in isolated atrial cardiomyocytes from CUG960 +dox mice. (A) Representative tracings of $\mathrm{Ca}^{2+}$ transient recordings during $1 \mathrm{~Hz}$ pacing and after exposure to $10 \mathrm{mM}$ caffeine, showing spontaneous $\mathrm{Ca}^{2+}$ waves in CUC960 +dox mice. Red arrows indicate calcium waves and yellow arrows indicate point of caffeine administration. Traces are drawn to scale with each other. Arbitrary units were used for F/FO. (B) $\mathrm{Ca}^{2+}$ waves incidence. (C) $\mathrm{Ca}{ }^{2+}$ transient amplitude. (D) Zoomed-in representative caffeine-induced $\mathrm{Ca}^{2+}$ transient tracings. (E) NCX activity calculated from the decay of the caffeine-induced transient and (F) SERCA activity calculated as the difference between the decay of the pacing-induced transient and the caffeine-induced transient in CUC960 +dox mice in comparison with MHCrtTA +dox controls and CUC960 +/off dox mice. $n=4$ animals per group and data points represent individual cardiomyocytes. Within each group, all cardiomyocytes from each mouse are depicted with distinct symbols. Data represent the mean $\pm \mathrm{SD}$. Ca ${ }^{2+}$ waves incidence was analyzed using Kruskal-Wallis 1-way ANOVA followed by Dunn's test for multiple comparisons. All other data were analyzed using the Generalized Estimating Equation function in SPSS. ${ }^{*} \mathrm{P}<0.05,{ }^{* *} \mathrm{P}<0.01$. CaT, $\mathrm{Ca}^{2+}$ transient; $\mathrm{NCX}, \mathrm{Na}^{+} / \mathrm{Ca}^{2+}$ exchanger; SERCA, sarco/endoplasmic reticulum calcium ATPase; dox, doxycycline.

and the ventricles. The cardiac conduction abnormalities, RNA foci formation, MBNL colocalization and alternative splicing defects were rescued in response to diminished CUGexp RNA expression.

Transcriptomic analysis of atrial and ventricular RNA isolated from CUG960 +dox mice revealed differential expression and alternative splicing changes in several genes involved in the regulation of cardiac ion transport (Figure 6 and Figure 7). CUG960 +dox mice exhibited increased expression of hyperpolarization-activated cyclic nucleotide-gated potassium channel 4 ( $\mathrm{Hcn} 4)$ and downregulation of gap junction protein $\alpha 5$ (Gja5) in atria (Figure $6 \mathrm{E}$ ). HCN4 contributes to the native pacemaker current in the heart and is involved in the regulation of heart rhythm. Mutations in HCN4 have been associated with sick sinus syndrome (45) and early-onset atrial fibrillation $(46,47)$. GJA5, a major gap junction protein in the atrial working myocardium, is responsible for intercellular propagation of the action potential. Somatic mutations in GJA5 have been linked to atrial fibrillation (48) and Gja5-knockout mice exhibit conduction defects and a higher predisposition to atrial arrhythmias $(49,50)$. CUG960 + dox mice displayed downregulation of sodium voltage-gated channel $\alpha$ subunit 10 (Scn10a) in ventricles (Figure 6E). The Scn10a gene encodes the $\alpha$ subunit of voltage-gated sodium channel Nav1.8, previously shown to be expressed in intracardiac neurons 
and cardiomyocytes and linked in genetic association studies with QRS interval (51) and QT interval (52) duration. In both atria and ventricles, CUG960 + dox mice showed strong reduction in Junctin mRNA levels (Figure 6E). Junctin is a SR transmembrane protein that forms a quaternary protein complex with the ryanodine receptor, calsequestrin, and triadin and is involved in the regulation of calcium release from the SR. Downregulation of Junctin levels is observed in heart failure (53) and knockout mouse models show aberrant calcium homeostasis leading to arrhythmias (54).

CUG960+dox mice exhibited alternative splicing defects in various genes involved in the regulation of the cardiac action potential (Figure 7E). The SCN5A gene encodes the major voltage-gated cardiac sodium channel Nav1.5, which is responsible for the influx of the $\mathrm{Na}+$ ions $\left(\mathrm{I}_{\mathrm{Na}}\right)$ contributing to the depolarization phase of the action potential. The voltage-sensing transmembrane region of SCN5A located in domain I, segments S3 and S4, is encoded by 2 developmentally regulated, mutually exclusive exons $6 \mathrm{~A}$ and $6 \mathrm{~B}$. While exon $6 \mathrm{~B}$ is predominantly included in the adult heart, $S C N 5 A$ mis-splicing resulting in inclusion of the fetal exon 6A is observed in DM1 (42). Previous studies have shown that inclusion of the fetal exon $6 \mathrm{~A}$ alters the electrophysiological properties of the channel resulting in slower channel kinetics and slower recovery from inactivation (55). Forced expression of the fetal isoform in adult mouse heart resulted in cardiac conduction defects and arrhythmias $(42,56)$. CUG960 +dox mice exhibited a switch from predominant inclusion of exon 6B to partial inclusion of fetal exon $6 \mathrm{~A}$ in both the atria and ventricles of the heart (Figure 7E). The voltage-gated potassium channel Kv4.3, encoded by the KCND3 gene, effluxes potassium ions, generating the transient outward current $\left(\mathrm{I}_{\text {tol }}\right)$ that contributes to the repolarization phase of the cardiac action potential. Kv channel interacting protein 2 (KChIP2), encoded by the KCNIP2 gene, serves as an auxiliary protein, which interacts with and modulates the activity of the Kv potassium channels such as Kv4.2 and Kv4.3. As observed in DM1 (40, 42), CUG960 +dox mice display skipping of Kcnd3 exon 6 and Kcnip2 exon 3 (Figure 7E). The alternatively spliced region in KCND3 encodes 19 amino acids at the C-terminal, including a threonine residue, which can be phosphorylated by PKC to regulate $\mathrm{I}_{\text {to }}(57)$, and the isoform lacking the exon shows faster inactivation, thereby contributing to lengthening of action potential duration (58). The KChIP2 isoform lacking exon 3 (KChIP2b) has been previously shown in in vitro studies to confer differential activation kinetics and recovery from inactivation for Kv4.3 channel gating (59).

We also observed alternative splicing changes in genes associated with the functions of calcium handling in the heart. RyR2, the predominant isoform of the ryanodine receptor family of ion channels expressed in the heart, regulates the flow of calcium ions out of the SR in cardiomyocytes. Abnormalities in RyR2 gating have been implicated in the development of atrial arrhythmias (60). In both the atria and the ventricles, CUG960 +dox mice exhibited skipping of exon 4 and 5 for Ryr2 (Figure 7E). The exclusion of $R y R 2$ exon 4 was recently demonstrated in iPSC-derived DM1 cardiomyocytes (61). However, the functional significance of the region encoded by this exon is currently unknown. CaMKII $\delta$ is a multifunctional Ser/Thr protein kinase involved in the regulation of calcium homeostasis by targeting various proteins involved in the calcium uptake and release such as the ryanodine receptors, phospholamban, and L-type calcium channels. The multiple Camk2d splice variants expressed in the heart can be distinguished based on the inclusion of exons 14,15, and 16, which alter the cellular localization and function of the protein (62). CUG960 + dox mice exhibited decreased inclusion of exons 14 and 15 and an overall shift in Camk2d splicing pattern toward the $\delta_{\mathrm{C}}$ isoform lacking exons 14, 15, and 16 (Figure 7E), which localizes to the cytoplasm and has been shown to phosphorylate RyR2 and phospholamban (63). Enhanced CaMKII $\delta$ phosphorylation of RyR2 has been shown to promote atrial arrhythmias (64).

Consistent with the observed transcriptomic alterations, we identified calcium-handling defects in atrial cardiomyocytes isolated from CUG960 + dox mice in comparison with controls. Increased diastolic calcium release from RyR2 in the form of calcium waves coupled with increased sodium influx though NCX can lead to delayed-after-depolarizations (DADs). DADs large enough to reach the excitation threshold for an action potential can trigger ectopic beats that contribute to arrhythmogenesis $(44,65)$.

In conclusion, we developed a CUGexp RNA-expressing mouse model of DM1 that recapitulated various physiological, cellular, and molecular aspects of cardiac DM1 pathogenesis. Importantly, these phenotypes are reversible in response to cessation of transgene expression, underscoring the therapeutic potential of targeting CUGexp RNA for reversal of disease features. Molecular transcriptomic analysis revealed dysregulation of several genes associated with the functions of ion transport and regulation of cardiomyocyte action potential at the level of mRNA abundance and alternative splicing. Isolated atrial cardiomyocytes from repeat RNA-expressing mice exhibited calcium-handling defects, which could potentially contribute to the observed 
arrhythmogenic phenotypes. Future work will entail detailed mechanistic investigations into individual candidate genes for their contribution to DM1 cardiac pathology.

\section{Methods}

Transgenic mice. TREDT960I mice were developed in an FVB background by pronuclear injection of linearized transgene using standard techniques as described (38). The TREDT960I transgene contains 960 interrupted CTG repeats in the context of a human genomic segment containing DMPK exons 11-15. The interrupted repeats were generated by ligating fragments containing 20 CTG repeats, which are separated by 5 nucleotide interruptions created by fusion of SalI and XhoI restriction sites. The repeats are stable for propagation in mice and bacteria. Southern blotting analysis confirmed that the number of repeats in the integrated transgene are stable across multiple generations (data not shown). The transgene is flanked by cHS4 insulator sequences on the $5^{\prime}$ and $3^{\prime}$ ends to prevent any effects associated with chromosomal integration sites (66). The TREDT960I mice are available from The Jackson Laboratory (stock no. 032050). MHC-rtTA transgenic mice (FVB/N-Tg(Myh6-rtTA)1Jam) expressing a codon-optimized variant of rtTA specifically in the heart were commercially obtained (RRID: MMRRC_010478) (39). Mice that were homozygous for TREDT960I and MHCrtTA transgenes were mated to mice homozygous for the TREDT960I transgene to obtain F1 progeny of bitransgenic mice homozygous for the TREDT960I transgene and hemizygous for MHCrtTA transgene, which were used for experiments. Mice hemizygous for MHCrtTA transgene that were used as controls were obtained by mating MHCrtTA hemizygous mice to wild type FVB mice. The study consisted of both male and female animals and is not gender specific. Mice were provided with dox-containing chow ( $2 \mathrm{~g} \mathrm{dox} / \mathrm{kg}$ chow, Bioserv) either beginning at PN1 through nursing dams or at 2 months or 6 months of age. Genomic DNA was isolated from tail clips using DirectPCR lysis reagent (Viagen Biotech) and evaluated by PCR using transgene-specific primers for genotypes. Primer sequences are provided in Supplemental Table 2.

RT-qPCR and RNA splicing. Total RNA was isolated from atrial and ventricular heart tissues using TRIzol reagent (15596-018; Invitrogen). cDNA was prepared from $1 \mu \mathrm{g}$ of DNase treated RNA (4368813; Thermo Fisher Scientific). Primers targeting human DMPK exons 12-14 were used to assay transgene expression by real-time qPCR. Mouse Rpl4 was used as internal control for normalization. PCR reactions were carried out on an Applied Biosystems 7500 Fast Real-Time PCR System using PowerUp SYBRGreen PCR master mix (Thermo Fisher Scientific). Relative expression levels were determined by the $2^{-\Delta \Delta C t}$ method. For analysis of alternative splicing events, primers annealing to flanking constitutive exons were designed. PCR products were resolved on a $5 \%$ polyacrylamide gel. Ethidium bromide stained RT-PCR bands were analyzed using Kodak Gel Logic 2000 and Carestream software. Percent spliced in (PSI) values were calculated using densitometry according to the equation: PSI $=100 \mathrm{X}$ [Inclusion band/(Inclusion band + Skipping band)]. Primer sequences are provided in Supplemental Table 2 and Supplemental Table 3. See complete unedited blots in the supplemental material.

Immunoblotting. Tissue protein extracts were prepared from atrial and ventricular heart tissues by homogenization followed by sonication in RIPA lysis buffer containing $1 \times$ Halt protease and phosphatase inhibitor cocktail (Thermo Fisher Scientific). Cellular debris was cleared by centrifugation $(16,000 \mathrm{~g}$ for 30 minutes at $\left.4^{\circ} \mathrm{C}\right)$. Protein lysates $(20-25 \mu \mathrm{g})$ were separated on $12 \%$ Tris-glycine SDS-PAGE gels and transferred to nitrocellulose membranes for Western blot analysis. Total protein was visualized by Ponceau S staining and membranes were incubated in anti-CELF1 (3B1, catalog 05-621, EMD Millipore, 1:500 dilution) or anti-GAPDH (14C10, catalog 2118, Cell Signaling Technology, 1:200,000 dilution) primary antibodies overnight at $4^{\circ} \mathrm{C}$. Membranes were washed 3 times in PBS (0.1\% Tween-20, Sigma) and incubated for 2 hours in HRP-conjugated goat anti-rabbit or goat anti-mouse (1:10,000, Jackson Immunoresearch) antibodies. After washing 3 times in PBST (0.1\% Tween-20), blots were imaged on a ChemiDoc XRS+ Imaging system (Bio-Rad). See complete unedited blots in the supplemental material.

Echocardiography. Echocardiographic examination of in vivo cardiac function and morphology was performed using a Vevo 2100 ultrasound machine equipped with a $40 \mathrm{MHz}$ transducer-MS550S (Visualsonics). For imaging, mice were anesthetized with $1.5 \%$ isoflurane and body temperature was maintained at $37^{\circ} \mathrm{C}$. M-mode images were acquired in the short-axis position at the level of papillary muscles for each animal. Three M-mode tracings were analyzed per animal using the Visualsonics VevoLab analysis package and values were averaged.

ECG. ECG data were obtained using a multichannel amplifier followed by conversion to digital signals for analysis. Surface ECG recordings were measured from 3 leads using a Rodent Surgical Monitor+ platform (Indus Instruments) and data were acquired using PowerLab 8/35 (AD Instruments). Mice were anesthetized 
using $2 \%$ isoflurane for data collection. Body temperature was monitored using a rectal probe. Data were collected for 1.5-2 minutes per mouse and ECG intervals were analyzed using LabChart software (AD Instruments). The QTc interval was calculated using Bazett's formula (67).

Intracardiac programmed electrical stimulation. Programmed intracardiac stimulation was performed to evaluate inducibility of atrial arrhythmias as previously described (68). Briefly, an incision was made to the right of the midline near the clavicle to access the right jugular vein for insertion of a $1.1 \mathrm{~F}$ octapolar catheter (EPR-800; Millar Instruments) into the right atrium and ventricle. Atrial arrhythmias were induced using a burst pacing protocol where a 2-second burst with a cycle length of $40 \mathrm{~ms}$ was applied followed by successive bursts each with $2 \mathrm{~ms}$ decrements down to a cycle length of $10 \mathrm{~ms}$. Pacing protocols were performed in triplicate and mice were considered positive for arrhythmia inducibility if pacing evoked arrhythmias lasting 1 second or longer, at least 2 out of 3 times. The incidence of inducible atrial arrhythmia was calculated as the percentage of arrhythmia-positive mice divided by the total number of mice studied.

FISH-IF. Combined FISH-IF was performed on frozen cardiac sections $(7 \mu \mathrm{m})$ using a modified protocol as previously described (8). Sections were fixed 30 minutes with $4 \% \mathrm{PFA} / 1 \times \mathrm{PBS}$ at room temperature, washed 5 times with $1 \times$ PBS for 2 minutes each followed by permeabilization in $2 \%$ prechilled acetone/1× PBS for 10 minutes. Sections were incubated with $30 \%$ formamide $/ 2 \times$ saline sodium citrate (SSC) for 30 minutes, hybridized with Tye-563-labeled (CAG5 LNA probes ( $0.5 \mathrm{ng} / \mu 1$, Exiqon-Qiagen) for 4.5 hours at $42^{\circ} \mathrm{C}$ in hybridization buffer ( $30 \%$ formamide, $2 \times \mathrm{SSC}, 0.02 \% \mathrm{BSA}, 66 \mu \mathrm{g} / \mathrm{mL}$ yeast tRNA, $2 \mathrm{mM}$ Vanadyl ribonucleoside complex), and washed for 30 minutes in $30 \%$ formamide $/ 2 \times \mathrm{SSC}$ at $42^{\circ} \mathrm{C}$. Sections were washed in $1 \times$ SSC for 30 minutes at room temperature followed by incubation in mouse monoclonal anti-MBNL1 (3A4, catalog sc-47740, Santa Cruz Biotechnology, 1:25 dilution) and anti-MBNL2 (3B4, catalog sc-136167, Santa Cruz Biotechnology, 1:25 dilution) antibodies overnight at $4^{\circ} \mathrm{C}$. Sections were washed 5 times in $1 \times$ PBS for 2 minutes, incubated in Alexa 488-labeled goat anti-mouse secondary antibody (1:1000, Invitrogen), washed 3 times with $1 \times$ PBS for 5 minutes each, incubated with DAPI $(0.5 \mu \mathrm{g} / \mathrm{mL})$, washed 5 times with $1 \times$ PBS for 2 minutes each, and mounted in Shandon Immu-Mount (Thermo Fisher Scientific). Images were acquired using a DeltaVision Elite (GE Healthcare) and processed using SoftWoRx software (GE Healthcare).

RNA-Seq analysis. RNA was isolated from atria and ventricles using the RNeasy Fibrous Tissue Mini Kit (QIAGEN). The Genomic and RNA Profiling Core first conducted sample quality checks using the NanoDrop spectrophotometer and Agilent Bioanalyzer 2100. The samples passing the following criteria were used for RNA-Seq: RNA integrity number $(\mathrm{RIN}) \geq 7.0, \mathrm{~A} 260 \mathrm{~nm} / \mathrm{A} 280 \mathrm{~nm} \geq 1.9$, A260nm/A230nm $\geq 1.2$, and $\mathrm{r} 28 \mathrm{~S} / 16 \mathrm{~S} \geq 1.5$. The Illumina TruSeq Stranded mRNA library preparation protocol was then used to generate cDNA libraries starting with 250 ng of total RNA. ERCC RNA Spike-In Controls were added to each sample according to the manufacturer's protocol. The resulting libraries were quantitated using the NanoDrop spectrophotometer and fragment size assessed with the Agilent Bioanalyzer. The pooled libraries were loaded onto a NovaSeq 6000 S4 flow cell and sequenced to a depth of approximately 150 million read pairs/ sample. A paired-end 150 cycle run was used to sequence the flow cell on a NovaSeq 6000 sequencing system. The data set has been deposited in the National Center for Biotechnology Information/Gene Expression Omnibus under accession number GSE164825. The raw fastq files were first quality checked using FastQC (version 0.11.8) software (http://www.bioinformatics.bbsrc.ac.uk/projects/fastqc/). Fastq files were aligned to mm10 mouse reference genome (GRCm38.39) and per-gene counts quantified by RSEM (69) (version 1.3.1) based on the gene annotation Mus_musculus.GRCm38.89.chr.gtf. Differential gene expression values were obtained using DESeq2 (70) (version 1.22.2). Gene expression differences were considered significant if passing the following criteria: adjusted $P$ value $<0.05, \log _{2}$ (fold change) $\geq 1.5$. For splicing analysis, reads were aligned to mm10 mouse reference genome (GRCm38.89) using STAR (71) (version 2.6.1a). Mapping percentage and sample details are provided in Supplemental Table 1. Based on the bam files generated from the alignment, splicing was quantified using rMATS (72) (version 4.0.2), which annotated and statistically analyzed 5 different kinds of splicing events (AS 5'SS, AS 3'SS, CE, MXE, and IR) using Mus_musculus. GRCm38.89.chr.gtf gene annotation. Splicing events were considered significant if passing the following criteria: reads $\geq 20$, FDR $\leq 0.05$, and $\triangle P S I \geq 0.15$. Gene ontology analysis was performed using DAVID (version 6.8). Enriched categories with $-\log _{10}(P$ value $) \geq 1.5(P$ value $\leq 0.032)$ were considered significant.

Cellular $\mathrm{Ca}^{2+}$ imaging. Atrial myocytes were isolated from adult mouse hearts using a retrograde Langendorff perfusion protocol as previously described (73). Cardiomyocytes that failed to follow pacing or did not have regular striations were not included in the study. Cells that were exhibiting pacemaker-like qualities with regular spontaneous depolarization or contractions were also not included in the study. Myocytes 
were incubated with $2 \mu \mathrm{M}$ Fluo-4-AM (Invitrogen) and imaging was performed in line scan mode on a Zeiss LSM 880 confocal microscope. Fluo-4-loaded myocytes were placed in a chamber equipped with parallel platinum electrodes and steady-state $\mathrm{Ca}^{2+}$ transients (CaTs) were induced by $1 \mathrm{~Hz}$ pacing $(5 \mathrm{~ms}$, $10 V)$. Pacing was then stopped for 20 seconds and $\mathrm{Ca}^{2+}$ sparks were counted. For measurements of total SR $\mathrm{Ca}^{2+}$ content (SR load), perfusate was switched to calcium-free Tyrode solution to block L-type calcium channel and prevent any new cellular entry of calcium. SR load was determined by application of $10 \mathrm{mM}$ caffeine after pacing. All transient measurements were normalized to baseline fluorescence (F0) as performed in previous studies (73). The incidence of $\mathrm{Ca}^{2+}$ waves was determined by calculating the number of cells displaying one or more noninduced CaTs (in the absence of pacing) divided by the total number of cells. SERCA activity was calculated as the difference between the decay rate of pacing-induced CaT (which reflects $\mathrm{Ca}^{2+}$ extrusion by combined activities of NCX and SERCA) and the decay rate of caffeine-induced CaT (which reflects $\mathrm{Ca}^{2+}$ extrusion by NCX only) as previously described (74). Data were analyzed using Clampfit and Image $(\mathrm{NIH})$ with a SparkMaster plugin.

Statistics. All quantitative experiments have at least 3 independent biological replicates. Results are presented as mean \pm SD. Statistical analyses for data sets were carried out using Prism software (version 8.0, GraphPad) and methods used are specified in figure legends. Statistical tests included 1-way ANOVA followed by Tukey's test for multiple comparisons; 2-way ANOVA followed by Tukey's test for multiple comparisons; Fisher's exact test; 2-tailed t test; and Kruskal-Wallis 1-way ANOVA followed by Dunn's test for multiple comparisons. Calcium imaging data were analyzed using the Generalized Estimating Equation function (SPSS version 25.0.0.0) where mouse number was set as the subject variable, cell number as within subject variable, and each respective calcium measurement as the dependent variable. A $P$ value of less than 0.05 was considered statistically significant.

Study approval. All mouse experiments were carried out in accordance with the Guide for the Care and Use of Laboratory Animals (National Academies Press, 2011) and approved by the Baylor College of Medicine Institutional Animal Care and Use Committee.

\section{Author contributions}

ANR designed the study, performed experiments, analyzed the data, and wrote the manuscript. HMC performed intracardiac pacing experiments, and TAW performed calcium imaging studies under the supervision of XHTW. ZX and XG performed computational analysis of sequencing reads from RNA-Seq data. TAC supervised and designed the study, analyzed the data, and wrote the manuscript. All authors contributed to the interpretation of results and production of the final manuscript.

\section{Acknowledgments}

ECG and echocardiography studies were performed at the Mouse Metabolic and Phenotyping Core at Baylor College of Medicine (BCM) supported by funds from the NIH (UM1HG006348, R01DK114356). FISH-IF images were acquired in the Integrated Microscopy Core at BCM, funded by the NIH (DK56338, and CA125123), CPRIT (RP150578, RP170719), the Dan L. Duncan Comprehensive Cancer Center, and the John S. Dunn Gulf Coast Consortium for Chemical Genomics. RNA library preparation and nextgeneration sequencing was performed at the Genomic and RNA Profiling Core at BCM under the direction of core director Daniel Kraushaar, and funding support from NIH (NIDDK-DK56338, NCI-CA125123, and 1S10OD02346901). This project was funded by grants from the NIH (R01HL045565, R01AR045653, R01AR060733, and R01HL147020) and the Muscular Dystrophy Association(MDA276796 and MDA602529) to TAC and a Wyck-Myotonic Dystrophy Foundation predoctoral fellowship grant to ANR. XHTW was funded by grants from the NIH (R01HL089598, R01HL091947, R01HL117641, and R01HL147108), HMC was funded by NIH grant F30HL140782, and TAW was funded by NIH grant T32HL139430-01A1. ZX was funded by NIH/NLM grant K01LM012877 and the Medical Research Foundation of Oregon. We thank Yuriana Aguilar for assisting with preparation of the revised manuscript. We thank Donnie Bundman for her work on transgene development. We also thank members of the Cooper lab for their valuable discussions and help throughout the project. Images were created with BioRender.

Address correspondence to: Thomas A. Cooper, Baylor College of Medicine, 1 Baylor Plaza, MS: BCM315, Houston, Texas 77030, USA. Phone: 713.798.5838; Email: tcooper@bcm.edu. 
1. Turner C, Hilton-Jones D. The myotonic dystrophies: diagnosis and management. J Neurol Neurosurg Psychiatry. 2010;81(4):358-367.

2. Brook JD, et al. Molecular basis of myotonic dystrophy: expansion of a trinucleotide (CTG) repeat at the 3' end of a transcript encoding a protein kinase family member. Cell. 1992;68(4):799-808.

3. Mahadevan M, et al. Myotonic dystrophy mutation: an unstable CTG repeat in the 3' untranslated region of the gene. Science. 1992;255(5049):1253-1255.

4. Sicot G, Gomes-Pereira M. RNA toxicity in human disease and animal models: from the uncovering of a new mechanism to the development of promising therapies. Biochim Biophys Acta. 2013;1832(9):1390-1409.

5. Davis BM, et al. Expansion of a CUG trinucleotide repeat in the 3 ' untranslated region of myotonic dystrophy protein kinase transcripts results in nuclear retention of transcripts. Proc Natl Acad Sci U S A. 1997;94(14):7388-7393.

6. Mankodi A, et al. Nuclear RNA foci in the heart in myotonic dystrophy. Circ Res. 2005;97(11):1152-1155

7. Miller JW, et al. Recruitment of human muscleblind proteins to (CUG) (n) expansions associated with myotonic dystrophy. EMBO J. 2000;19(17):4439-4448.

8. Mankodi A, et al. Muscleblind localizes to nuclear foci of aberrant RNA in myotonic dystrophy types 1 and 2. Hum Mol Genet 2001;10(19):2165-2170.

9. Kuyumcu-Martinez NM, et al. Increased steady-state levels of CUGBP1 in myotonic dystrophy 1 are due to PKC-mediated hyperphosphorylation. Mol Cell. 2007;28(1):68-78.

10. Kalsotra A, et al. The Mef2 transcription network is disrupted in myotonic dystrophy heart tissue, dramatically altering miRNA and mRNA expression. Cell Rep. 2014;6(2):336-345.

11. Kalsotra A, et al. A postnatal switch of CELF and MBNL proteins reprograms alternative splicing in the developing heart. Proc Natl Acad Sci U S A. 2008;105(51):20333-20338.

12. Wang ET, et al. Antagonistic regulation of mRNA expression and splicing by CELF and MBNL proteins. Genome Res. $2015 ; 25(6): 858-871$

13. Batra R, et al. Loss of MBNL leads to disruption of developmentally regulated alternative polyadenylation in RNA-mediated disease. Mol Cell. 2014;56(2):311-322.

14. Thomas JD, et al. Disrupted prenatal RNA processing and myogenesis in congenital myotonic dystrophy. Genes Dev. 2017;31(11):1122-1133.

15. Wang ET, et al. Transcriptome alterations in myotonic dystrophy skeletal muscle and heart. Hum Mol Genet. 2018;28(8):1312-1321.

16. de Die-Smulders CE, et al. Age and causes of death in adult-onset myotonic dystrophy. Brain. 1998;121 (Pt 8):1557-1563.

17. Petri $\mathrm{H}$, et al. High prevalence of cardiac involvement in patients with myotonic dystrophy type 1 : a cross-sectional study. Int $J$ Cardiol. 2014;174(1):31-36.

18. Caroline C-N, et al. Association between mutation size and cardiac involvement in myotonic dystrophy type 1. Circ Cardiovasc Genet. 2017;10(3):001526.

19. Hideki I, et al. Cardiac conduction disorders as markers of cardiac events in myotonic dystrophy type 1. J Am Heart Assoc. 2020;9(17):015709.

20. Johnson NE, et al. Parent-reported multi-national study of the impact of congenital and childhood onset myotonic dystrophy. Dev Med Child Neurol. 2016;58(7):698-705.

21. Johnson NE, et al. Disease burden and functional outcomes in congenital myotonic dystrophy: a cross-sectional study. Neurology. 2016;87(2):160-167.

22. Groh WJ, et al. Electrocardiographic abnormalities and sudden death in myotonic dystrophy type 1. N Engl J Med. 2008;358(25):2688-2697.

23. Park KM, et al. Prolonged corrected QT interval in patients with myotonic dystrophy type 1. J Clin Neurol. 2013;9(3):186-191.

24. Petri H, et al. Cardiac manifestations of myotonic dystrophy type 1. Int J Cardiol. 2012;160(2):82-88.

25. Brembilla-Perrot B, et al. Atrial flutter or fibrillation is the most frequent and life-threatening arrhythmia in myotonic dystrophy. Pacing Clin Electrophysiol. 2014;37(3):329-335.

26. Nguyen $\mathrm{HH}$, et al. Pathology of the cardiac conduction system in myotonic dystrophy: a study of 12 cases. J Am Coll Cardiol. 1988;11(3):662-671.

27. Uemura N, et al. Electrophysiological and histological abnormalities of the heart in myotonic dystrophy. Am Heart J. 1973;86(5):616-624

28. Nazarian S, et al. QRS prolongation in myotonic muscular dystrophy and diffuse fibrosis on cardiac magnetic resonance. Magn Reson Med. 2010;64(1):107-114.

29. Bhakta D, et al. Prevalence of structural cardiac abnormalities in patients with myotonic dystrophy type I. Am Heart J. 2004;147(2):224-227.

30. Bhakta D, et al. Increased mortality with left ventricular systolic dysfunction and heart failure in adults with myotonic dystrophy type 1. Am Heart J. 2010;160(6):1137-1141.

31. Lee KY, et al. Compound loss of muscleblind-like function in myotonic dystrophy. EMBO Mol Med. 2013;5(12):1887-1900.

32. Koshelev M, et al. Heart-specific overexpression of CUGBP1 reproduces functional and molecular abnormalities of myotonic dystrophy type 1. Hum Mol Genet. 2010;19(6):1066-1075.

33. Mahadevan MS, et al. Reversible model of RNA toxicity and cardiac conduction defects in myotonic dystrophy. Nat Genet. 2006;38(9):1066-1070

34. Yadava RS,et al. MBNL1 overexpression is not sufficient to rescue the phenotypes in a mouse model of RNA toxicity. Hum Mol Genet. 2019;28(14):2330-2338.

35. Tylock KM, et al. Biophysical mechanisms for QRS- and QTc-interval prolongation in mice with cardiac expression of expanded CUG-repeat RNA. J Gen Physiol. 2020;152(2):201912450.

36. Wang G-S, et al. Elevation of RNA-binding protein CUGBP1 is an early event in an inducible heart-specific mouse model of myotonic dystrophy. J Clin Invest. 2007;117(10):2802-2811.

37. Algalarrondo V, et al. Abnormal sodium current properties contribute to cardiac electrical and contractile dysfunction in a mouse model of myotonic dystrophy type 1. Neuromuscul Disord. 2015;25(4):308-320.

38. Morriss GR, et al. Mechanisms of skeletal muscle wasting in a mouse model for myotonic dystrophy type 1 . Hum Mol Genet. 
2018;27(16):2789-2804.

39. Valencik ML, McDonald JA. Codon optimization markedly improves doxycycline regulated gene expression in the mouse heart. Transgenic Res. 2001;10(3):269-275.

40. Misra $\mathrm{C}$ et al. Aberrant expression of a non-muscle RBFOX2 isoform triggers cardiac conduction defects in myotonic dystrophy. Dev Cell. 2020;52(6):748-763.

41. Giudice J, et al. Alternative splicing regulates vesicular trafficking genes in cardiomyocytes during postnatal heart development. Nat Commun. 2014;5:3603.

42. Freyermuth F, et al. Splicing misregulation of SCN5A contributes to cardiac-conduction delay and heart arrhythmia in myotonic dystrophy. Nat. Commun. 2016;7:11067.

43. Dixon DM, et al. Loss of muscleblind-like 1 results in cardiac pathology and persistence of embryonic splice isoforms. Sci Rep. 2015;5(1):9042.

44. Landstrom AP, et al. Calcium signaling and cardiac arrhythmias. Circ Res. 2017;120(12):1969-1993.

45. Eyal N, et al. Point mutation in the HCN4 cardiac ion channel pore affecting synthesis, trafficking, and functional expression is associated with familial asymptomatic sinus bradycardia. Circulation. 2007;116(5):463-470.

46. Macri V, et al. A novel trafficking-defective HCN4 mutation is associated with early-onset atrial fibrillation. Heart Rhythm 2014;11(6):1055-1062.

47. Schweizer $\mathrm{P}$, et al. Altered HCN4 channel C-linker interaction is associated with familial tachycardia-bradycardia syndrome and atrial fibrillation. Eur Heart J. 2012;102(3):2675-2775.

48. Gollob MH, et al. Somatic mutations in the connexin 40 gene (GJA5) in atrial fibrillation. N Engl J Med. 2006;354(25):2677-2688.

49. Kirchhoff S, et al. Reduced cardiac conduction velocity and predisposition to arrhythmias in connexin 40-deficient mice. Curr Biol. 1998;8(5):299-302.

50. Suveer B, et al. Altered right atrial excitation and propagation in connexin40 knockout mice. Circulation. 2005;112(15):2245-2253

51. Sotoodehnia N, et al. Common variants in 22 loci are associated with QRS duration and cardiac ventricular conduction Nat Genet. 2010;42(12):1068-1076.

52. Abou Ziki MD, et al. Deleterious protein-altering mutations in the SCN10A voltage-gated sodium channel gene are associated with prolonged QT. Clin Genet. 2018;93(4):741-751.

53. Gergs U, et al. On the role of junctin in cardiac Ca2+ handling, contractility, and heart failure. Am J Physiol Heart Circ Physiol. 2007;293(1):H728-H734.

54. Qunying Y, et al. Sarcoplasmic reticulum calcium overloading in junctin deficiency enhances cardiac contractility but increases ventricular automaticity. Circulation. 2007;115(3):300-309.

55. Onkal R, et al. Alternative splicing of Nav1.5: an electrophysiological comparison of 'neonatal' and 'adult' isoforms and critical involvement of a lysine residue. J Cell Physiol. 2008;216(3):716-726.

56. Pang PD, et al. CRISPR -mediated expression of the fetal Scn5a isoform in adult mice causes conduction defects and arrhythmias. J Am Heart Assoc. 2018;7(19):010393.

57. Kong W, et al. Isolation and characterization of the human gene encoding Ito: further diversity by alternative mRNA splicing Am J Physiol. 1998;275(6):H1963-H1970.

58. Xie C, et al. Closed-state inactivation in Kv4.3 isoforms is differentially modulated by protein kinase C. Am J Physiol Cell Physiol. 2009;297(5):C1236-C1248.

59. Takimoto K, et al. Palmitoylation of KChIP splicing variants is required for efficient cell surface expression of Kv4.3 channels J Biol Chem. 2002;277(30):26904-26911.

60. Dobrev D, et al. The ryanodine receptor channel as a molecular motif in atrial fibrillation: pathophysiological and therapeutic implications. Cardiovasc Res. 2010;89(4):734-743.

61. Kim EY, et al. Distinct pathological signatures in human cellular models of myotonic dystrophy subtypes. JCI Insight. 2019;4(6):122686

62. Gray C, Heller Brown J. CaMKIIdelta subtypes: localization and function. Front Pharmacol. 2014;5:15.

63. Tong $\mathrm{Z}$, et al. The $\delta \mathrm{C}$ isoform of $\mathrm{CaMKII}$ is activated in cardiac hypertrophy and induces dilated cardiomyopathy and heart failure. Circ Res. 2003;92(8):912-919.

64. Chelu MG, et al. Calmodulin kinase II-mediated sarcoplasmic reticulum Ca2+ leak promotes atrial fibrillation in mice. J Clin Invest. 2009;119(7):1940-1951.

65. Niels V, et al. Enhanced sarcoplasmic reticulum Ca2+ leak and increased $\mathrm{Na}+\mathrm{Ca} 2+$ exchanger function underlie delayed afterdepolarizations in patients with chronic atrial fibrillation. Circulation. 2012;125(17):2059-2070.

66. Chung JH, et al. A 5' element of the chicken beta-globin domain serves as an insulator in human erythroid cells and protects against position effect in Drosophila. Cell. 1993;74(3):505-514.

67. Bazett HC. An analysis of the time-relations of electrocardiograms. Ann Noninvasive Electrocardiol. 1997;2(2):177-194

68. Li N, Wehrens XHT. Programmed electrical stimulation in mice. J Vis Exp. 2010;(39):1730

69. Li B, Dewey CN. RSEM: accurate transcript quantification from RNA-Seq data with or without a reference genome. $B M C$ Bioinformatics. 2011;12(1):323.

70. Love MI, et al. Moderated estimation of fold change and dispersion for RNA-seq data with DESeq2. Genome Biol. 2014;15(12):550.

71. Dobin A, et al. STAR: ultrafast universal RNA-seq aligner. Bioinformatics. 2012;29(1):15-21.

72. Shen S, et al. rMATS: robust and flexible detection of differential alternative splicing from replicate RNA-Seq data. Proc Natl Acad Sci U S A. 2014;111(51):E5593-E5601.

73. Wagner E, et al. Analysis of tubular membrane networks in cardiac myocytes from atria and ventricles. J Vis Exp. 2014 ;(92):51823.

74. Niels V, et al. Cellular and molecular mechanisms of atrial arrhythmogenesis in patients with paroxysmal atrial fibrillation.

Circulation. 2014;129(2):145-156 